Article

\title{
Insights from Bacteroides Species in Children with Type 1 Diabetes
}

\author{
José Matos $^{1,2}$, Isabel Matos ${ }^{1,2}$, Manuela Calha ${ }^{3}$, Pedro Santos ${ }^{1,2}$, Isabel Duarte ${ }^{4} \mathbb{D}^{\text {, }}$, Yameric Cardoso ${ }^{1}$ \\ and Maria Leonor Faleiro $1,2,5, *$ (i)
}

check for updates

Citation: Matos, J.; Matos, I.;

Calha, M.; Santos, P.; Duarte, I.;

Cardoso, Y.; Faleiro, M.L. Insights from Bacteroides Species in Children with Type 1 Diabetes. Microorganisms 2021, 9, 1436. https://doi.org/ 10.3390/microorganisms 9071436

Academic Editor: Khosrow Adeli

Received: 4 June 2021

Accepted: 29 June 2021

Published: 2 July 2021

Publisher's Note: MDPI stays neutral with regard to jurisdictional claims in published maps and institutional affiliations.

Copyright: (c) 2021 by the authors. Licensee MDPI, Basel, Switzerland. This article is an open access article distributed under the terms and conditions of the Creative Commons Attribution (CC BY) license (https:/ / creativecommons.org/licenses/by/ $4.0 /)$.
1 Faculdade de Ciências e Tecnologia, C8, Campus de Gambelas, Universidade do Algarve, 8005-139 Faro, Portugal; jose.j.matos@gmail.com (J.M.); isabel.csc.matos@gmail.com (I.M.); pedroeenterprise@gmail.com (P.S.); yamericsilva@gmail.com (Y.C.)

2 Algarve Biomedical Center, Research Institute, 8005-139 Faro, Portugal

3 Unidade de Diabetologia, Centro Hospitalar Universitário do Algarve, 8000-386 Faro, Portugal; mmcalha@gmail.com

4 CINTESIS-Center for Health Technology and Services Research, Universidade do Algarve, 8005-139 Faro, Portugal; giduarte@ualg.pt

5 Champalimaud Research Program, Champalimaud Centre for the Unknown, 1400-038 Lisbon, Portugal

* Correspondence: mfaleiro@ualg.pt

\begin{abstract}
In our previous study the enrichment of the intestinal proteome of type 1 diabetes (T1D) children with Bacteroides proteins was observed, which led us to our current study that aimed to isolate and characterize Bacteroides species from fecal samples of T1D and control children. Repetitive sequence-based PCR (rep-PCR) was used for typing the isolated Bacteroides species. The antibiotic susceptibility and mucinolytic activity of the isolates was determined. The quantification of specific bacterial groups in the fecal samples was determined by qPCR. The ability to adhere and invade the human colonic cell line HT29-MTX-E12 of strains of P. dorei, B. uniformis and P. distasonis was determined and their whole genome sequencing was performed. The results showed similar numbers of Bacteroides species in T1D and control samples, but unique Bacteroides species and a higher recovery of $P$. distasonis from T1D samples was observed. Rep-PCR grouped the different Bacteroides species, but no discrimination by origin was achieved. T1D children showed a significant increase in Proteobacteria and a depletion in Lactobacillus sp. All tested P. dorei, B. uniformis and P. distasonis were able to adhere to HT29-MTX-E12 cells but significant differences $(p<0.05)$ in the ability to invade was observed. The highest ability to invade was exhibited by P. distasonis PtF D14MH1 and P. dorei PtFD16P1, while B. uniformis strains were unable to invade. The damage to tight junctions was also observed. The presence of Lactobacillus sp. inhibited the invasion ability of P. distasonis PtF D14MH1 but not P. dorei PtFD16P1. Sequences of agonist peptides of the human natural preproinsulin and the insulin B chain insB:9-23 peptide mimics were identified. The results reported in our study stresses the continued efforts required to clarify the link between T1D and gut microbiota.
\end{abstract}

Keywords: type 1 diabetes; Phocaeicola dorei; Parabacteroides distasonis; Bacteroides uniformis; invasion; phage; molecular mimicry

\section{Introduction}

Type 1 diabetes (T1D) is characterized by a gradual pancreatic $\beta$-cell destruction culminating with insulin deficiency causing a hyperglycemic state, which in the mediumto long-term will cause vascular damage (neuropathy), affecting vision (retinopathy) and kidney function (nephropathy). The burden of genetic heritage in T1D is well recognized. However, the increase in incidence cannot be explained only by a genetic boost. The probability that environmental factors contribute to trigger the disease is currently gaining broader acceptance [1,2]. Among the environmental factors that may be implicated in this triggering event are the exposure of genetic susceptible individuals at early age to gluten 
containing food, viral upper respiratory tract infections, and exposure to deficient innate immune activator [3-6].

The disturbance of the gut microbiome in children at risk of developing T1D has been extensively investigated worldwide [7-16]. In contrast the microbiome of children with established diabetes is still poorly studied [7,14,17-20]. In the study of Alkanani et al. [7] the gut microbiome of new-onset patients and seropositive subjects from USA with two to four autoantibodies showed a higher $(p=0.01)$ median percentage abundance of Bacteroides $(35.62 \%)$ (21 subjects in the group of new-onset patients, and 16 in the seropositive group) in comparison with individuals with just one autoantibody (6.37), (12 subjects in the newonset patients group, and five in the seropositive group). Additionally, De Goffau et al. [21] reported higher abundance of the genus Bacteroides in children with $\beta$-cell autoimmunity who participated in the Finnish Dietary Intervention trial for Prevention of Type 1 Diabetes (FINDIA), and the Trial to Reduce IDMM in the Genetically at Risk (TRIGR) studies performed in Finland. The study conducted by Murri et al. [17] showed that Spanish children with established T1D carried more Bacteroidetes in comparison with healthy children. The higher abundance of sequences belonging to Bacteroides in the gut of Spanish children with T1D was also reported in the study of Leiva-Gea et al. [20], where they observed an enrichment of $72.21 \%$ in T1D cases in contrast to healthy controls that reached $58.45 \%$.

Geographical factors may considerably influence the gut microbiome [12]. For example, in Finland, in the city of Turku, it was reported that Bacteroides dorei (currently classified as Phocaeicola dorei [22]), dominates the gut of children at risk of developing T1D [8], whereas in the cities of Tampere and Oulu the increase of B. dorei was not observed [23]. Instead, these authors observed a dominant OTU, grouping B. dorei and B. vulgatus, that were inversely associated with following islet autoimmunity. Furthermore, in their study another Bacteroides sp., B. caccae was inversely associated with islet autoimmunity [23]. The control children in the Cinek et al. study [23] were enriched with an OTU assigned to Bifidobacterium bifidum. Another example of geographical differences regarding the composition of T1D children microbiome is the study conducted in the Northern area of China where children newly diagnosed with T1D ( $<6$ months) showed an intestinal microbiota enriched with the genus Blautia that was also correlated with the levels of HbA1c, T1D autoantibodies, and titers of tyrosine phosphatase autoantibodies (IA-2) [19]. Furthermore, in the study of Kostic et al. [13] it was reported that the abundance of the genus Blautia was correlated with serum glucose levels in children with T1D.

Portuguese children with established T1D showed a metaproteome enriched with proteins originated from Eubacterium rectale (currently Agathobacter rectalis), Faecalibacterium prausnitzii, B. dorei and $B$. uniformis, in contrast with the control metaproteome that was enriched in proteins from Bifidobacterium adolescentis, B. longum subsp. infantis, Ruminococcus, Collinsella aerofaciens, Coprococcus comes, and Clostridium sp. [18]. However, the qPCR evaluation of Bacteroides sp. and Bifidobacterium sp. were similar between case and control children, suggesting that the intestinal environment was not affecting the bacterial population, instead modulating its proteome [18]. Altogether, it is important to stress that the gut microbiota in children, either at risk of T1D or with established T1D, shows a dysbiosis, and the genus Bacteroides may have some role in the development of the disease.

This study aimed to characterize Bacteroides species from fecal samples of T1D children and control children from Portugal (Algarve region), and analyze their characteristics regarding antibiotic susceptibility, mucinolytic activity, its potential to disturb intestinal epithelial cells, and also analyze their genomic characteristics that may be linked with the development of T1D. 


\section{Materials and Methods}

\subsection{Fecal Samples}

Seventeen fecal samples from T1D children, aged $9.53 \pm 1.88$ were obtained (eight female and seven male). The control group included 15 healthy T1D siblings and children with no T1D history in the immediate family, aged $8.20 \pm 3.00$. All children followed a regular diet ( $>50 \%$ of meat, eggs, vegetables, fruit and milk of regular origin). For the majority of children, sports were practiced twice a week or three times a week, only four T1D children reported not to practice any sports. T1D children were medicated exclusively with insulin. The haemoglobin level (HbA1c) in diabetic children was $8.76 \pm 1.50$. Samples from each child were collected, immediately transported to the laboratory in cold storage conditions, and directly surveyed. Antibiotic treatment, diagnosis of infectious diseases, and hospitalization up to 3 months before the start of the study were exclusion criteria.

The current study was approved by the Ethics Committee of the Centro Hospitalar Universitario do Algarve, and informed consent was obtained from the parents of the children enrolled in the study.

\subsection{Bacteria and Culture Conditions}

To isolate Bacteroides species from fecal samples, the culture medium bacteroides vulgatus selective agar (BVSA) was used [24]. About $0.2-0.3 \mathrm{~g}$ of stool specimen was collected to a sterile $2 \mathrm{~mL}$ eppendorf, and the feces were resuspended in $1 \mathrm{~mL}$ of Phosphate Buffered Saline (PBS). After homogenization, each sample was centrifuged at low speed, $700 \times g$ for $5 \mathrm{~min}$. Next, $500 \mu \mathrm{L}$ of the supernatant was collected to a new eppendorf and serial dilutions were prepared. A volume of $100 \mu \mathrm{L}$ of the dilutions $10^{-3}$ and $10^{-4}$ were inoculated in BVSA, and the incubation was achieved using an anaerobic jar with an anaerobic sachet (AnaeroGen, Oxoid, Basingstock, UK) at $37{ }^{\circ} \mathrm{C}$ for $3-4$ days. Afterwards, characteristic colonies (black, shiny colonies with a dark halo zone) were counted, and representative colonies of each morphological group were transferred to brain-heart infusion (VWR) supplemented with hemin $(0.1 \%, v / v)$ and L-cysteine $(0.1 \%, w / v$, Sigma-Aldrich, St. Louis, $\mathrm{MO}, \mathrm{USA})(\mathrm{BHI}+\mathrm{H})$ to obtain pure cultures. The incubation was conducted at $37{ }^{\circ} \mathrm{C}$ for $48 \mathrm{~h}$. Pure cultures were maintained in BHI supplemented with $25 \%(v / v)$ glycerol at $-80{ }^{\circ} \mathrm{C}$.

\subsection{DNA Extraction}

The extraction of DNA from stool samples was performed using the kit QIAmp DNA Stool Mini Kit (QIAGEN, Hilden, Germany) according to the manufacturer's instructions. The stool quantity used for DNA extraction was $220 \mathrm{mg}$. The extracted DNA was eluted in $200 \mu \mathrm{L}$ of AE buffer and maintained at $-20^{\circ} \mathrm{C}$ until use.

The DNA from bacterial cultures was extracted using the Wizard Genomic DNA Purification Kit (PROMEGA) or the GES method [25].

\subsection{Identification of Bacteroides Species by $16 S$ rRNA Gene Sequencing}

Molecular identification of Bacteroides species was performed by sequencing the $16 S$ $r R N A$ gene using the primers pair 27F and 1492R [26]. The Bacteroides isolates were cultured in $\mathrm{BHI}+\mathrm{H}$ for $48 \mathrm{~h}$ at $37^{\circ} \mathrm{C}$ under anaerobic conditions. Genomic DNA was extracted as described above. The polymerase chain reactions (PCR) amplifications were carried out with the DFS-Taq DNA Polymerase (Bioron GmbH, Römerberg, Germany). Purified PCR products were sequenced with the 1492R primer [27] at the Molecular Biology laboratory of the Centro de Ciências do Mar (CCMar) using standard Sanger sequencing procedures.

The $16 S$ rRNA gene sequences were analyzed, and those $\geq 1200 \mathrm{bp}$ were selected for further analysis. The editing of the sequences was conducted using Bioedit (version 7.2.5). The identification of the closest matches to sequence queries was performed using the Blast algorithm of the National Center for Biotechnology Information (NCBI) [27]. The Ribosomal Database Project [28] was also used for the determination of the isolates at the species/strain level. 


\subsection{Characterization of Bacteroides Species by Repetitive Sequence-Based PCR}

The Bacteroides isolates were analyzed by rep-PCR with BOX A1R primer and ERIC1R and ERIC2 primers $[29,30]$. The PCR reactions were performed with Platinum Taq polymerase (Invitrogen, Eugene, OR, USA).

The PCR amplicons $(25 \mu \mathrm{L})$ were separated by gel electrophoresis on $1.5 \%$ Agarose (Lonza, Risingevej, Denmark) gel in TAE $1 \times$ buffer at $100 \mathrm{~V}$ during $6 \mathrm{~h}$. The 100 and $500 \mathrm{bp}$ markers (Invitrogen) were used. Gels were stained with ethidium bromide for $30 \mathrm{~min}$ and visualized under UV light using the DC 290 Kodac camera.

Analysis of the gel banding patterns was performed using GelCompare II (Biomérieux, Lisbon, Portugal) and dendograms were generated using Pearson correlation similarity coefficient with optimization of $1 \%$, and the cluster analysis was performed using the unweighted pair group method using arithmetic averages (UPGMA) clustering method.

\section{6. $q P C R$}

The quantification of specific bacterial groups (Firmicutes, Bacteroidetes, Bacteroides, P. dorei, Bifidobacterium sp., Clostridium cluster IV, Enterococcus sp., Lactobacillus sp. Proteobacteria and Prevotella sp.) was determined by quantitative real-time PCR (qPCR). The specific primers used are indicated in Table S1. Reactions were performed using SsoFastTM kit Eva Green Supermix (BioRad, Hercules, CA, USA), $0.5 \mu \mathrm{M}$ of forward and reverse primer and 100 ng of DNA on a CFX96 qPCR thermal cycler (Bio-Rad Laboratories, Hercules, CA, USA). All PCR reactions were carried out in duplicate, and the PCR conditions are shown in Table S2. Reactions containing all the components except template DNA were included as negative controls. The denaturation step occurred at $95{ }^{\circ} \mathrm{C}$, for $10 \mathrm{~min}$. The number of cycles was identical for all target groups ( 45 cycles). The melting curve was performed by increasing the temperature $0.5^{\circ} \mathrm{C}$ each $2 \mathrm{~s}$ from $72{ }^{\circ} \mathrm{C}$ to $95^{\circ} \mathrm{C}$.

Standard curves were generated using plasmid DNA ( $\mathrm{pCR}$ TM 2.1-TOPO) with inserted $16 S r R N A$ gene of each bacterium. The cloning process was achieved using competent Escherichia coli MACH1 (Invitrogen) following the instructions of the vector manufacturer (Invitrogen). Four replicates of the plasmid DNA dilutions in order to obtain the number of copies that ranged from $10^{10}-10$ were prepared. Data are expressed as $\log _{10}$ DNA copies/g feces.

\subsection{Antibiotic Susceptibility}

The determination of antibiotic susceptibility was achieved as previously described [31,32]. Briefly, Bacteroides strains were grown on BHI $+\mathrm{H}$ and incubated in an Anaerobic jar (AnaeroGen, Oxoid) at $37^{\circ} \mathrm{C}$ for $24 \mathrm{~h}$. Bacterial suspensions were prepared in $0.9 \%$ saline to a density of McFarland 1 . The disc diffusion assays were performed using Brucella blood agar plates supplemented with hemin $(0.1 \%)$ and vitamin K1 (1\%). The antibiotic discs (Oxoid) were amoxycillin-clavulanate (20/10 $\mu \mathrm{g})$ (AMC), clindamycin $(10 \mu \mathrm{g})(\mathrm{DA})$, imipinem $(10 \mu \mathrm{g})(\mathrm{IMP})$, moxifloxacin $(5 \mu \mathrm{g})(\mathrm{MXF})$, piperacillin-tazobactam $(30 / 6 \mu \mathrm{g})(\mathrm{TZP})$ and metronidazole $(5 \mu \mathrm{g})(\mathrm{MTZ})$. The plates were incubated in anaerobic conditions at $37^{\circ} \mathrm{C}$ for $24 \mathrm{~h}$. Six discs (three per plate) were used. The susceptibility zone diameter provisional breakpoints previously suggested [31,32] were followed: amoxycillin-clavulanate $(\geq 15 \mathrm{~mm})$, clindamycin $(\geq 25 \mathrm{~mm})$, imipenem $(\geq 29 \mathrm{~mm})$, moxifloxacin ( $\geq 19 \mathrm{~mm})$, piperacillin-tazobactam $(\geq 25 \mathrm{~mm}$ ) and metronidazole $(\geq 24 \mathrm{~mm})$. Next, the results for Imipinem and Metronidazole were confirmed by Epsilon test according to manufacturer's procedure (Biomérieux, Portugal). The breakpoints were established according to EUCAST [33].

\subsection{Mucinolytic Activity}

The ability of several strains of $P$. dorei isolated from T1D and control children to use mucin as sole carbon source was evaluated by growing the bacterial strains in a defined medium [24] supplemented with 1\% porcine gastric mucin (PGM) (Sigma, Madrid, Spain) previously dialyzed with SnakeSkin ${ }^{\circledR}$ Dialysis Tubing membrane (cut off: $10 \mathrm{kDa}$ ) at 
$4{ }^{\circ} \mathrm{C}$. As control the bacterial growth was performed in the mentioned defined medium supplemented with glucose as sole carbon source $(0.5 \%, w / v)$ and in $\mathrm{BHI}+\mathrm{H}$ medium. The growth was followed by changes in optical densities $\left(\mathrm{OD}_{600 \mathrm{~nm}}\right)$ in microplates. For this $180 \mu \mathrm{L}$ of medium were distributed in each well, and each well was inoculated with $20 \mu \mathrm{L}$ of the bacterial culture previously grown in the tested medium for $24-48 \mathrm{~h}$ in anaerobic conditions. Wells with non-inoculated medium were used as blanks. To assure anaerobic conditions, $30 \mu \mathrm{L}$ of sterile paraffin oil were added into each well, and the incubation was performed in an anaerobe jar (BioMérieux) with an anaerobic sachet (Oxoid) at $37^{\circ} \mathrm{C}$ for $48 \mathrm{~h}$. The optical readings were performed on a microplate reader (Tecan Infinite M200). Four biological replicates were used. The reference strain of P. dorei DSM 17855 and the strain of P. vulgatus PtF D2P1 were included.

The mucinolytic activity was evaluated as described by Yesilkaya et al. [34]. The bacterial strains were grown in Defined medium with $1 \%(w / v)$ mucin and $1 \%$ agar (DMa). As control, non-inoculated DMa plates were used. The inoculated culture plates were incubated in anaerobic jars during $48 \mathrm{~h}$ at $37^{\circ} \mathrm{C}$. Next, the culture plates were stained with $0.1 \%(w / v)$ black amid in acetic acid 3.5 $\mathrm{M}$ for $30 \mathrm{~min}$ and were decolorized with acetic acid 1.2 M. Escherichia coli D3 (isolated from a T1D child) and E. coli C1 (isolated from a healthy child) were included as controls. Mucinolytic activity zones were observed around the bacterial growth.

\subsection{Whole Genome Sequencing}

Genomic DNA of strains P. dorei PtFD1P5, PtFD8M1, PtFD16P1, PtFD16M14, PtFSb6, PtFSb8, PtFC1P2, B. uniformis PtFD3Pch2, PtFSb3P5 and P. distasonis PtFD14MH1 for whole genome sequencing was isolated from $2 \mathrm{~mL}$ of the bacterial culture prepared as described above. The DNA was extracted using Wizard Genomic DNA Purification Kit (Promega, Madison, WI, USA), according to the manufacturer's protocol with a modification that included an additional step of lysis with sterile acid-washed glass beads $(425-600 \mu \mathrm{m})$ (Sigma Life Science, Saint Louis, MO, USA) comprising 1/3 of the volume of the bacterial pellet. The mixture was sonicated for $1 \mathrm{~min}$ using the bead beater (Next Advance, Troy, NY, USA).

DNA integrity was analyzed by electrophoresis using a $0.75 \%(w / v)$ agarose gel (Lonza, Copenhagen, Denmark). DNA quantification was performed by using the fluorescence-based Qubit dsDNA BR Assay Kit (Invitrogen, Eugene, OR, USA). Each bacterial genomic DNA was dissolved in $10 \mathrm{mM}$ Tris- $\mathrm{HCl}$ buffer at concentrations ranging from 2.7 to $5.0 \mu \mathrm{g} / \mu \mathrm{L}$ for Standard Whole Genome Service by Illumina next-generation sequencing provided by MicrobesNG (http:/ / www.microbesng.uk, accessed on 2 October 2018) which is supported by the BBSRC (grant number BB/L024209/1). At Microbes NG Genomic DNA libraries were prepared using the Nextera XT Library Prep Kit (Illumina, San Diego, CA, USA) following the manufacturer's protocol with minor modifications, namely input DNA was increased 2-fold, and PCR elongation time increased to $45 \mathrm{~s}$. DNA quantification and library preparation were performed on a Hamilton Microlab STAR automated liquid handling system (Hamilton Bonaduz AG, Bonaduz, Switzerland). Pooled libraries were quantified using the Kapa Biosystems Library Quantification Kit for Illumina. Libraries were sequenced using Illumina sequencers (HiSeq) using a $250 \mathrm{bp}$ paired end protocol.

Reads were adapter trimmed using Trimmomatic 0.30 with a sliding window quality cutoff of Q15 [35]. De novo assembly was performed using SPAdes version 3.7 [36], and contigs were annotated using Prokka 1.11 [37]. The scaffolding of the contigs was achieved using scaffold builder [38].

The circular genome maps were generated using CGView server Beta (version 1.7) [39]. The tracks GC Skrew, GC Content and Prokka Annotation were added [37]. In Prokka Annotation the default parameters for bacterial genome were used.

Whole genome sequence reads were deposited in the NCBI SRA under BioProject ID PRJNA687382. The GenBank assembly accessions are GCA_018588045.1,GCA_018588075.1, 
GCA_018588055.1,GCA_018588025.1,GCA_018588125.1,GCA_018588105.1,GCA_018588135.1, GCA_018704025.1, GCA_018704075 and GCA_018587995.1

\subsection{Typing}

The typing of the whole genome sequenced strains P. dorei PtFD1P5, PtFD8M1, PtFD16P1, PtFD16M14, PtFSb6, PtFSb8, PtFC1P2, B. uniformis PtFD3Pch2, PtFSb3P5 and P. distasonis PtFD14MH1 was performed by using the multilocus sequencing typing (MLST) using 30 genes listed in Table S3 following the recommendations of Chun et al. and Munoz et al. [40,41]. The selected genes are involved in the housekeeping apparatus and the central metabolic pathways of the bacterial cell. The sequence alignment was performed using Clustal Omega [42], and the phylogenetic analysis and tree construction (using concatenated aligned sequences), was conducted with the software MEGA X [43]. As reference, the sequences of the listed genes of the strains P. dorei DSM 17855, CL03T12C01, CL02T12C06, CL02T00C15; HS1 L3 B 079, HS2 L2 B045b, HS1 L1B010, B. uniformis ATCC 8492 and P. distasonis ATCC 8503 were used.

\subsection{Prophage and CRISPR-Cas Systems Detection}

The P. dorei, B. uniformis and P. distasonis genomes were analyzed with PHASTER (PHAge Search Tool Enhanced Release) (http:/ / phaster.ca/, accessed on 4 June 2021) [44] to search for potential prophage sequences. CRISPRs (clustered Regularly Interspaced Short Palindromic Repeats) and Cas (CRISPR-associated) proteins were searched using the CRISPRCasFinder [45].

\subsection{Identification of Resistance, Pathogenicity Potential and Molecular Mimicry}

In order to identify acquired antimicrobial resistance genes, the software CARD/RGI was used [46]. The identification of the potential pathogenicity to human host was conducted using the software PathogenFinder 1.1 [47]. The molecular mimicry identification was conducted using BLASTP (version 2.2.26) through SEED Viewer (version 2.0) by searching the presence of the insulin $B$ chain insB:9-23 peptide mimics (SHLVEALYLVCGERG) described in P. distasonis 33B, which was found in the Human Gut Microbiome [48], and the two microbial altered peptides ligands (MVWGPDPLYV and RQFGPDWIVA) of the natural preproinsulin (ALWGPDPAAA) reported in Bacteroides fragilis/thetaiotaomicron and Clostridium asparagiforme, respectively [49].

\subsection{Cell Culture Assays}

The human colonic cell line HT29-MTX-E12 (ECACC 12040401) (Sigma-Aldrich, European Collection of Authenticated Cell Cultures, Porton Down, Salisbury, UK) was routinely cultured in Dulbecco's Modified Eagle Medium (DMEM, Sigma-Aldrich, St. Louis, MO, USA) supplemented with 10\% $(v / v)$ fetal bovine serum (FBS), $1 \%(v / v)$ non-essential amino acids, and antibiotics (Penicillin/Streptomycin (PEN-STREP), 5000 units $/ \mathrm{mL}$ Penicillin and $5000 \mu \mathrm{g} / \mathrm{mL}$ Streptomycin) (Gibco, Carlsbad, CA, USA) (DMEM complete). Cultures were incubated at $37{ }^{\circ} \mathrm{C}$ in a humidified $5 \%(v / v) \mathrm{CO}_{2}$ atmosphere and grown until $80-90 \%$ confluence before being sub-cultured and used between passages 52 to 60 .

For adhesion, invasion, and evaluation of the damage to the tight junctions assays, the cell tissue culture was performed for 21 days post seeding to reach mature HT29-MTX-E12 cells. Cells were seeded in 24-well culture plates at concentration of $4.0 \times 10^{4}$ cells per well. The culture medium was changed every two days for the 21 days of the differentiation period. In the last medium change no antibiotics were added to the medium.

\subsubsection{Adherence Assay}

The evaluation of the adhesion ability of strains of P. dorei, B. uniformis and P. distasonis to HT29-MTX-E12 intestinal cells was performed as previously described by Gagnon et al. [50] with some modifications. The bacterial cultures were grown in $\mathrm{BHI}+\mathrm{H}$ in anaerobic conditions during $24 \mathrm{~h}$ at $37^{\circ} \mathrm{C}$. The previous bacterial culture was used to inoculate at 
$10^{7} \mathrm{CFU} / \mathrm{mL}$ the HT29-MTX-E12 cells prepared as previously described. The tissue culture plates were then incubated at $37^{\circ} \mathrm{C}$ under anaerobic conditions for $30 \mathrm{~min}$. After this time interval the non-adherent bacteria were eliminated by washing with (PBS) (twice). Subsequently, the cell line with adherent bacteria was treated with $250 \mu \mathrm{L}$ Trypsin-EDTA (Sigma-Aldrich, St. Louis, USA per well and incubated at $37^{\circ} \mathrm{C}$ for $10 \mathrm{~min}$. To inactivate the Trypsin-EDTA, DMEM supplemented with 10\% $(v / v)$ of FBS was added to each well. From this suspension, serial decimal dilutions were performed in PBS, and then each dilution was inoculated on $\mathrm{BHI}+\mathrm{H}$ agar plates. The inoculated plates were incubated under anaerobic conditions at $37^{\circ} \mathrm{C}$ for $48 \mathrm{~h}$.

The adhesion results are expressed as the percentage of the number of adherent bacterial cells relative to the total number of bacteria used on the assay.

\subsubsection{Invasion Assay}

The evaluation of the invasion ability of strains of $P$. dorei, B. uniformis and P. distasonis to HT29-MTX-E12 intestinal cells was performed as previously described by Gagnon et al. [50] with some modifications. Briefly, the bacterial cultures were grown in $\mathrm{BHI}+\mathrm{H}$ in anaerobic conditions for $24 \mathrm{~h}$ at $37^{\circ} \mathrm{C}$. The bacteria were then inoculated at $10^{7} \mathrm{CFU} / \mathrm{mL}$ into the plates with the HT29-MTX-E12 cells prepared as previously described. The tissue cell plates were then incubated at $37^{\circ} \mathrm{C}$ under anaerobic conditions for $4 \mathrm{~h}$. The infected cell lines were washed with PBS (twice) and exposed to $250 \mu \mathrm{L}$ DMEM medium containing $150 \mu \mathrm{g} / \mathrm{mL}$ of Gentamicin (Sigma-Aldrich, Germany) per well for $1 \mathrm{~h}$ at $37^{\circ} \mathrm{C}$ to eliminate any non-invasive bacteria. A new washing step was carried out with PBS, and $250 \mu \mathrm{L}$ Trypsin-EDTA was added to each well with further incubation at $37^{\circ} \mathrm{C}$ for $10 \mathrm{~min}$. The cell lines were then exposed to $0.1 \%(v / v)$ Triton X-100 (Merck, Darmstadt, Germany) for $10 \mathrm{~min}$ at $37^{\circ} \mathrm{C}$ to permeabilize the cells allowing the release of invasive bacteria. From this suspension, serial decimal dilutions were prepared, and the bacterial counts were performed as described above.

The results of the invasion ability are expressed as the percentage of the number of invasive bacteria relative to the total bacteria used in the assay.

\subsubsection{Ability of Bacteroides Species to Impair Epithelial Integrity}

The capacity of $P$. dorei, $B$. uniformis and P. distasonis strains to cause alterations to the epithelial integrity of the cell line HT29-MTX-E12 was evaluated as previously described in [50] with slight modifications. The cells were seeded in wells containing a sterile coverslip, Ø 13 and $1.5 \mathrm{~mm}$ thickness (VWR, Radnor, Philadelphia, PA, USA). Bacterial suspensions at a concentration of $10^{7} \mathrm{CFU} / \mathrm{mL}$ were transferred to the epithelial intestinal cells after the differentiation period of 21 days. The cell line was exposed to the bacterial cells during $4 \mathrm{~h}$ at $37^{\circ} \mathrm{C}$ under anaerobic conditions. The infected cells were then washed with PBS 3 times and fixed with 3.7\% $(v / v)$ formaldehyde (Labscan Analytical Sciences, Gliwice, Poland) for $15 \mathrm{~min}$. Three new washing steps with PBS were performed. The cell line was then permeabilized with $0.5 \%$ Triton X-100 (Merk, Darmstadt, Germany) for $10 \mathrm{~min}$, followed by 3 washing steps with PBS. The cells were then blocked for non-specific binding with $3 \%(w / v)$ bovine serum albumin (Merk, Germany) at $4{ }^{\circ} \mathrm{C}$ for $1 \mathrm{~h}$. Thereafter the cells were incubated during 20 min with tetramethylrhodamine B isothiocyanate-phalloidin (TRITC-phalloidin) (Sigma-Aldrich, Hamburg, Germany) (diluted 1:200 from an initial solution of $0.1 \mathrm{mg} / \mathrm{mL}$ ). Afterwards the cell line was exposed for $3 \mathrm{~min}$ to $4^{\prime}, 6^{\prime}$-diamidine2-phenylindole (DAPI) (Sigma-Aldrich, Germany) (diluted 1:200 of an initial solution of $14.3 \mathrm{mM}$ ). Two final washes were carried out with PBS. The assays were performed using three biological and two technical replicates.

Each coverslip was then mounted inverted on a microscope slide with a drop of Fluromount (Sigma-Aldrich, Germany). The observation of the cell lines was performed using the microscope Axio Imager Z2 (Zeiss, Oberkochen, Germany). 
2.13.4. Exposure of Intestinal Epithelial Cells to the Bacteroides Metabolome (Secretome)

The evaluation of the effect of the Bacteroides metabolome on the viability of the cell line HT29-MTX-E12 was performed using the Vibrant MTT cell proliferation assay kit (Molecular Probes, Invitrogen, Eugene, OR, USA) following the manufacturer instructions. Briefly, the bacterial cells were grown in $\mathrm{BHI}+\mathrm{H}$ at $37^{\circ} \mathrm{C}$ for $24 \mathrm{~h}$ under anaerobic conditions. Thereafter the bacterial suspensions were centrifuged $\left(5000 \times g, 10 \mathrm{~min}, 4^{\circ} \mathrm{C}\right)$ and the supernatant was collected and filtered $(0.2 \mu \mathrm{m}$ pore diameter, Whatman, GE Healthcare, Buckinghamshire, UK). The supernatant samples were stored at $-20^{\circ} \mathrm{C}$ until use.

HT29-MTX-E12 cells required were seeded in a 96-well microplate (8000 cells per well) and incubated for $48 \mathrm{~h}$ at $37^{\circ} \mathrm{C}$ in a $5 \%(v / v) \mathrm{CO}_{2}$ humidified atmosphere. Afterwards DMEM complete was discarded and replaced with DMEM complete supplemented with the bacterial supernatant at different percentages (10, 25 and 50\% [v/v]). Three biological and 4 technical replicates were used. The $\mathrm{pH}$ value of the different combinations was $7.8 \pm 0.11$ for the $10 \%(v / v)$ mix, $7.53 \pm 0.12$ for $25 \%(v / v)$ and for $50 \%(v / v)$ the pH was $7.21 \pm 0.26$. The cell line was exposed to the bacterial supernatant for $24 \mathrm{~h}$. After this time interval, the DMEM medium with the supernatant was replaced with DMEM complete, and the MTT solution was added to each well. The cell line was then incubated at $37^{\circ} \mathrm{C}$ in a $5 \%(v / v) \mathrm{CO}_{2}$ humidified atmosphere for $4 \mathrm{~h}$. Thereafter an SDS-HCl solution was gently added into each well followed by an incubation for $4 \mathrm{~h}$ at $37^{\circ} \mathrm{C}$ in a $5 \%(v / v) \mathrm{CO}_{2}$ humidified atmosphere. Finally, each well was homogenized to eliminate any precipitate and the absorbance value was determined at $570 \mathrm{~nm}$ using a microplate reader (Tecan Infinite, M200, Männedorf, Switzerland).

\subsubsection{Invasion Ability of Bacteroides Species in the Presence of Lactobacillus casei}

The ability of strains of $P$. dorei, B. uniformis and P. distasonis in the presence of Lactobacillus casei DSM 20011 to invade HT29-MTX-E12 intestinal cells was performed as described above. The L. casei DSM 20011 was grown on Man, Rogosa and Sharpe (MRS) medium under microaerophilic conditions for $24 \mathrm{~h}$ at $37^{\circ} \mathrm{C}$. Bacteroides and L. casei were inoculated at $10^{7} \mathrm{CFU} / \mathrm{mL}$ to the intestinal epithelial cells. The incubation conditions and recovery of bacterial cells that were able to invade were performed as described in Section 2.13.2, except that L. casei cells counts were conducted using the MRS medium under microaerophilic conditions, whereas Bacteroides spp. were performed in BHI + H supplemented with vancomycin $(15 \mu \mathrm{g} / \mathrm{mL})$ and kanamycin $(200 \mu \mathrm{g} / \mathrm{mL})$ and the incubation was conducted in anaerobic conditions.

The results are expressed as the percentage of the number of invasive bacteria relative to the total bacteria used in the assay.

\subsection{Statistical Analysis}

Data was analyzed for statistical significance by one or two-way ANOVA with the software SPSS (version 25) (Inc., Chicago, IL, USA). Statistical significance was considered at $p<0.05$; when the analysis was statistically significant, the Tukey's post hoc test was performed. Violin plots on bacterial abundances with qPCR results were performed using GraphPad Prism (version 9.0) (GraphPad Software, San Diego, CA, USA).

\section{Results}

\subsection{Counts and Diversity of Bacteroides Species}

The number of Bacteroides species recovered from culture achieved similar values ( $p>0.05$ ) between T1D and control samples; $6.64 \pm 0.74 \log _{10}$ CFU/g feces for T1D children and $6.40 \pm 0.06 \log _{10} \mathrm{CFU} / \mathrm{g}$ feces for control children. 
The different Bacteroides species isolated from the fecal samples of the two groups are illustrated in Figure 1. Several Bacteroides species were only isolated from T1D samples, namely the species B. graminisolvens, B. cellulosilyticus, B. finegoldii, B. stercoris, B. eggerthii and $B$. fragilis that achieved $3.45 \%$ each and none from controls. The recovery of $P$. dore $i$ in culture was attained from only three T1D samples reaching $10.34 \%$, whereas in controls $P$. dorei was recovered from four samples achieving $18.18 \%$. In T1D samples several Bacteroides species were recovered at similar percentages to $P$. dorei, such as $B$. uniformis and P. vulgatus $(10.34 \%)$. The percentage of recovery of B. ovatus and B. xylanisolvens was similar for both groups $(13.79 \%)$.

(a)

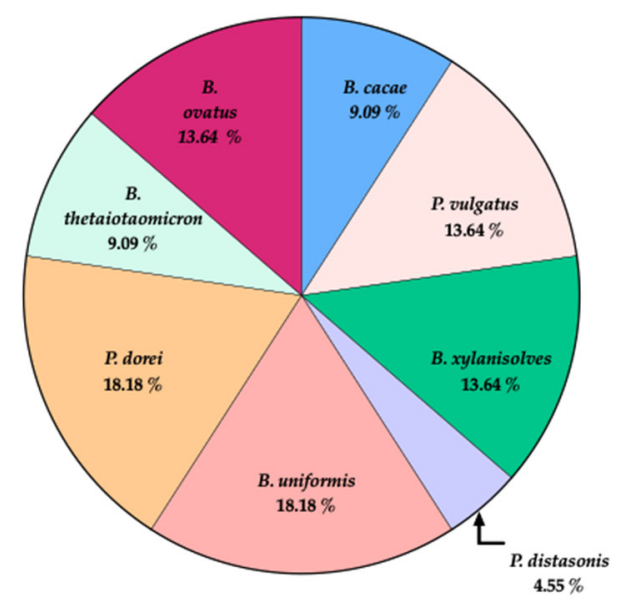

T1D

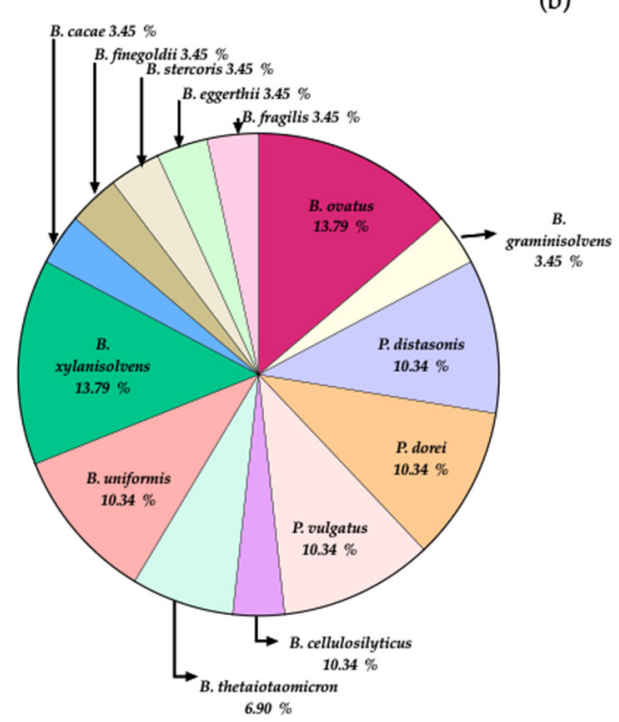

Figure 1. Bacteroides species recovered in culture from (a) control and (b) T1D fecal samples.

Overall, the core of Bacteroides species recovered from the two groups, besides P. dorei, includes the species B. ovatus, $P$. vulgatus, B. uniformis and B. xylanisolvens. Interestingly the recovery of $P$. distasonis was higher from T1D samples (10.34\%) in comparison with control samples $(4.55 \%)$.

The load of the targeted bacterial groups in the fecal samples of T1D and control children determined by qPCR is illustrated in Figure 2. Similar amounts $(p>0.05$, and values presented as $\log _{10}$ copy number/g feces) for both groups were found for Firmicutes (control $9.63 \pm 1.09$, T1D $9.39 \pm 0.93$ ), Bacteroidetes (control $8.35 \pm 0.40$, T1D $8.31 \pm 0.51$ ), Bacteroides sp. (control 7.10 \pm 0.32 , T1D $6.97 \pm 0.52$ ), P. dorei (control $3.97 \pm 1.52$, T1D $4.21 \pm 1.43$ ), Bifidobacterium sp. (control $7.30 \pm 0.60$, T1D $7.22 \pm 0.51$ ), Clostridium cluster IV (control $6.80 \pm 0.55$, T1D $6.70 \pm 0.68$ ), and Enterococcus sp. (control $4.14 \pm 0.43$, T1D 4.15 $\pm 0.38)$. However, T1D children carried lower numbers of Lactobacillus sp. $(p<0.05)$ in comparison with control children, but a higher abundance $(p<0.05)$ of Prevotella sp. and Proteobacteria was observed in their fecal samples. 


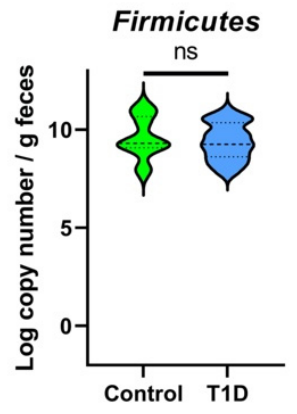

Bacteroidetes

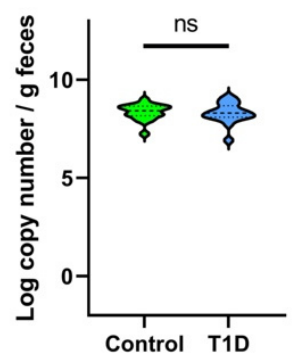

Bifidobacterium sp.
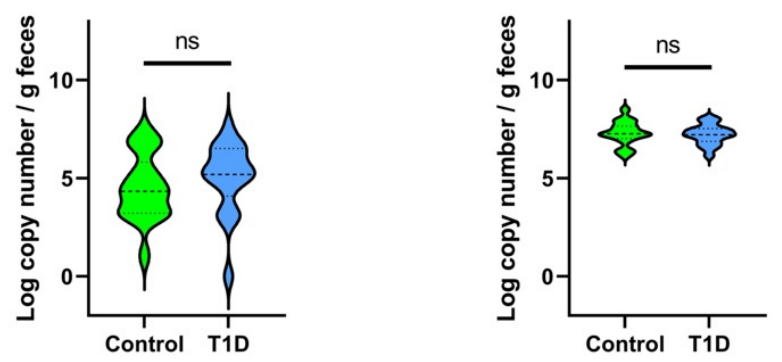

Enterococcus sp.

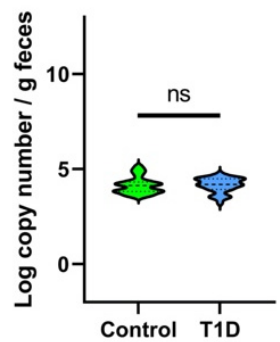

Prevotella sp.

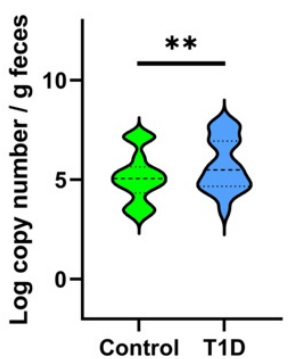

Bacteroides

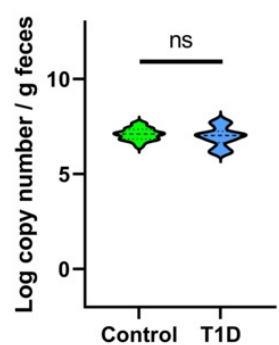

Clostridium Cluster IV

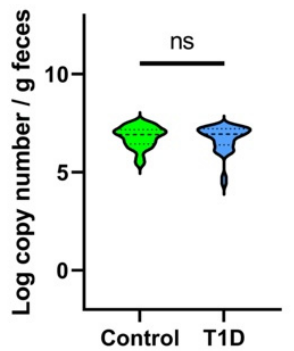

Lactobacillus sp.

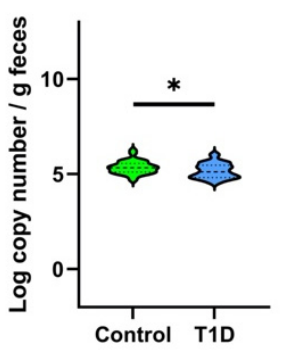

Proteobacteria

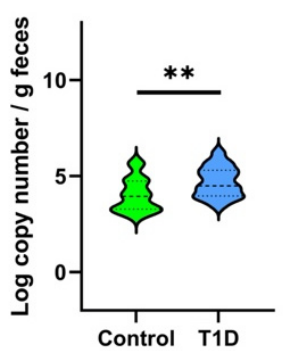

Figure 2. Violin plots reporting the qPCR quantification of fecal bacterial groups in T1D and control children ( $n=17$ participants per group). $p$-values were computed using the Mann-Whitney U test, ${ }^{*} p<0.05,{ }^{* *} p<0.001, \mathrm{~ns}-$ not significant.

\subsection{Antibiotic Susceptibility}

Bacteroides species are the most implicated anaerobic bacteria in infections, and resistance to antibiotics varies among the different species and also across geographic regions [31,51]. To evaluate the antibiotic susceptibility, a set of Bacteroides isolates was selected and their susceptibility was determined by disc diffusion including the antibiotics amoxycillin-clavulanate, clindamycin, imipenem, moxifloxacin, piperacillin-tazobactam, and metronidazole. The tested antibiotics are used in the treatment of infections caused by 
anaerobic bacteria $[31-33,51]$. The results of the antibiotic susceptibility are summarized in Table S4. Regarding the susceptibility of $P$. dorei strains for the antibiotic amoxycillinclavulanate, two resistant strains in T1D group were observed and in the control group three resistant strains were detected. More than half of the $P$. dorei strains were resistant to clindamycin $(58 \%)$, and even in the control group three strains were resistant to this antibiotic. For imipenem about $92 \%$ of the $P$. dorei strains were resistant, including the strains from the control group where just 1 strain was susceptible to IMP. In contrast $83 \%$ of the $P$. dorei strains were susceptible to moxifloxacin, and it was on the control group that it was found two resistant strains. For piperacillin-tazobactam more than half of the strains were resistant (58\%) and they were observed in both groups. The strains of B. uniformis and the isolate of $P$. distasonis were susceptible to amoxycillin-clavulanate, and just two strains were susceptible to clindamycin. For imipinem only two strains of $B$. uniformis were susceptible. In contrast just one strain of $B$. uniformis was resistant to moxifloxacin belonging to the control group. The resistance to piperacillin-tazobactam was detected in one strain of B. uniformis isolated from control children (B. uniformis PtFC3M3). All the strains of P. dorei, B. uniformis and P. distasonis were susceptible to metronidazole (Table S4).

The epsilon test for the antibiotic imipinem evidenced that all tested $P$. dorei strains were susceptible to this antibiotic, in contrast with the disc diffusion assay for which only one $P$. dorei strain was susceptible (Table S5). The strain of B. uniformis PtF Sb3P5 and P. distasonis PtFD14MH1 that according to the disc diffusion test were resistant, using the epsilon these strains are susceptible. The epsilon test for MTZ $(0.016-256 \mu \mathrm{g} / \mathrm{mL})$ evidenced MIC values between $0.0195-0.250 \mu \mathrm{g} / \mathrm{mL}$ (breakpoint $\mathrm{S} \leq 4 \mathrm{R} \geq 4 \mathrm{mg} / \mathrm{L}$ ) confirming the disc diffusion results; all tested strains of P. dorei, B. uniformis, and P. distasonis PtFD14MH1 were susceptible to this antibiotic.

The results of the in silico analysis of the genome of the strains P. dorei PtF D1P5, PtF D8M1, PtF D16P1, PtF D16M14, PtF Sb6, PtF Sb8, PtFC1P2, B. uniformis PtF D3Pch2, PtF Sb3P5 and P. distasonis PtF D14MH1 for the identification of resistance genes using the CARD/RGI are summarized in Table S6. The adeF gene that belongs to the antimicrobial resistance (AMR) gene family of resistance-nodulation cell division (RND) antibiotic efflux, and confers resistance to fluoroquinolone and tetracycline antibiotics, was detected on all strains, and in some strains more than one copy was present. Interestingly, the strains $P$. dorei PtF D16P1 and PtF D16M14 only carry this resistance gene.

The mef (En2) gene that belongs to the AMR gene familiy of major facilitator superfamily (MFS) antibiotic efflux pump that confers resistance to macrolide antibiotic was detected in four strains of P. dorei; PtF D1P5, PtF D8M1, PtF Sb6 and PtF Sb8. The tetQ gene that belongs to the AMR gene family tetracycline-resistant ribosomal protection protein was identified in six strains; five strains of P. dorei (PtF D1P5, PtF D8M1,PtF Sb6 PtF Sb8, PtF C1P2) and in the B. uniformis strain PtF D3Pch2. The gene ErmF that belongs to the Erm $23 \mathrm{~S}$ ribosomal RNA methyltransferase family was detected in two strains of $P$. dorei, $\mathrm{PtF}$ $\mathrm{D} 8 \mathrm{M} 1$ and PtF Sb8 and in the B. uniformis strain PtF Sb3P5. P. distasonis PtF D14MH1 carries a gene that encodes a beta-lactamase of the CfxA family, CfxA2 that has been found in Prevotella intermedia [52]. The strain of $B$. uniformis $\mathrm{PtF}$ Sb3P5 carries two beta-lactamase genes, namely OXA-347and CblA-1 that is found in B. uniformis and is species specific [53,54]. Single nucleotide polymorphisms (SNPs) were not detected.

\subsection{Mucinolytic Activity}

In defined medium with glucose as sole carbon source, all $P$. dorei strains and the strain of $P$. vulgatus PtFD2P1 reached a four fold growth in this medium. In contrast, the fold change in the defined medium with PGM was only one fold for all tested strains (Figure S1). The growth in the rich medium BHI attained a two fold increase, and the strain of $P$. dorei PtF D1P5 was the one that reached slighty higher than two fold in BHI (Figure S1).

Mucin degradation was revealed by the amidoblack staining of solidified defined medium with PGM where the cultures of the the strains of P. dorei and P. vulgatus caused the appearance of clear zones demonstrating mucinolytic activity. The cultures of $E$. coli 
strain D3 and C1 showed no mucin degradation zones around their growth evidencing their inability to degrade mucin (data not shown).

\subsection{Repetitive Based Sequence PCR}

The PCR patterns generated either with BOX primer (BOX-PCR) or the ERIC primers (ERIC-PCR) distinguished the different Bacteroides species, P. dorei, P. vulgatus and P. distasonis isolates (Figure S2). The cluster analysis of BOX-PCR fingerprints grouped the $P$. dorei isolates in three groups; the reference strain $P$. dorei DSM 17855 grouped with $P$. dorei PtFSb8 (80.8\% similarity) and the second cluster included the main isolates (PtFD16P1, PtFD16M14, PtFSb19Pp7, PtFSb6, PtFD1P5, PtFD1P20, PtFD1P21 and PtFD8M1). Two $P$. dorei isolates (PtFC1P2 and PtFC1M8) grouped with P. vulgatus (73.4\% similarity).

The cluster analysis based on the BOX-PCR for B. uniformis identified two main clusters; the first included the isolates B. uniformis PtFD3P28, PtFSb3P5 and Sb13P5 (97.3 and $96.6 \%$ ) and the second included the isolate B. uniformis PtFD6P31with $87.6 \%$ similarity between them. The isolate $B$. uniformis PtFC7NB15 showed low relatedness with the two main clusters (33.1\%). Two other isolates of B. uniformis (PtFC3M3 and PtFC6Pp6) clustered with $B$. thetaiotaomicron with $66.9 \%$ similarity. Interestingly, the isolate B. uniformis D3Pch2 showed a singular singleton clustering with B. ovatus (59.1\%) (Figure S2A).

Analysis of banding patterns produced using the BOX rep-PCR for B. xylanisolvens formed two main clusters, one includes the isolates from a unique T1D child, PtFD6Phid, PtFD6M1, PtFD6M10, PtFD6P1, PtFD6P5 achieving 99\% similarity, indicating that they can constitute a single strain. This grouping for these B. xylanisolvens isolates was also observed using the ERIC rep-PCR (Figure S3). The second cluster of B. xylanisolvens includes the isolates PtFD4M1, PtFD13MH1, PtFD3P4, PtFD3M6, PtFD3P6, PtFSb3P12, PtFSb3M1 showing high similarity (73.3\%). Other B. xylanisolvens isolates clustered with other Bacteroides species, namely the isolate B. xylanisolvens PtFD4P14 clustered with two isolates of B. ovatus with $93.2 \%$ relatedness, B. xylanisolvens PtFSb9MH2 clustered with the isolate of $B$. fragilis PtFD13MH3 with $98.1 \%$ similarity and the isolate B. xylanisolvens PtFSb2Phid clustered with P. vulgatus PtF D17P19 showing 78.1\% similarity.

The BOX rep-PCR banding pattern analysis for $B$. thetaiotaomicron grouped the isolates PtF C6P14, PtFC6P14Smo achieving 97.9\% similarity and the isolate PtFD4P1 was related with this cluster with $88 \%$ similarity. Two other B. thetaiotaomicron isolates PtFD4M3 and PtFD4 M11 (92.2\%) were related with the other isolates at $85.1 \%$ similarity. The isolate $B$. thetaiotaomicron PtFD2P14 was a singleton showing $61.0 \%$ similarity with the other isolates. Two other B. thetaiotaomicron singletons were identified, the isolate PtFD4P11 and the isolate PtFC2Phid (Figure S2A).

The cluster analysis of ERIC-PCR fingerprints, as stated above, discriminated the Bacteroides isolates by species (Figure S2B). The $P$. dorei isolates were grouped differently from the BOX-PCR producing three groups (Figure S2B). The first group includes the isolates P. dorei PtFC1M8, PtFD1P20, PtFD1P20, PtFSb8, the second includes the isolates PtFD16P1 and PtFD16M14, and the third group includes the isolates PtFD1P5 and PtFSb6. The $P$. dorei PtFC1P2 forms a singleton with a very low number of bands produced by ERIC primers, evidencing $71.9 \%$ similarity with B. stercoris and B. finegoldii (Figure S2B). A very lower number of bands produced by ERIC-PCR primers is observed in two B. uniformis isolates; PtFC3M3 and PtFC7NB15. Singletons in ERIC-PCR cluster analysis were identified in several Bacteroides species, namely B. thetaiotaomicron PtFD2P14, B. uniformis PtFD3Pch2 and PtFC6Pp6, B. xylanisolvens PtFD4P14 and PtFD4M1, B. ovatus PtFC4P5 and PtFC5M11 (Figure S2B).

\subsection{Interaction with Intestinal Epithelial Cells}

The interaction of $P$. dorei strains with intestinal epithelial cells was evaluated using the cell line HT-29-MTX-E12. The ability of $P$. dorei, B. uniformis and P. distasonis to adhere and invade the HT-29-MTX cells is illustrated in Figure 3. All tested strains of P. dorei, B. uniformis and the strain of P. distasonis PtFD14MH1 were equally $(p>0.05)$ able to adhere 
to HT-29-MTX cells, except the P. dorei strain PtFD16P1 and PtF Sb8 that showed a lower adherence $(p<0.05)$ (Figure 3A). The highest ability to invade was displayed by the strain of $P$. distasonis PtFD14MH1 $(p<0.05)$. The invasion ability of $P$. dorei PtF D16P1 and PtF Sb8 was higher $(p<0.05)$ in comparison with the other P. dorei strains. The B. uniformis strains PtF D3Pch2 and PtF Sb3P5 were not able to invade HT-29-MTX cells (Figure 3B).
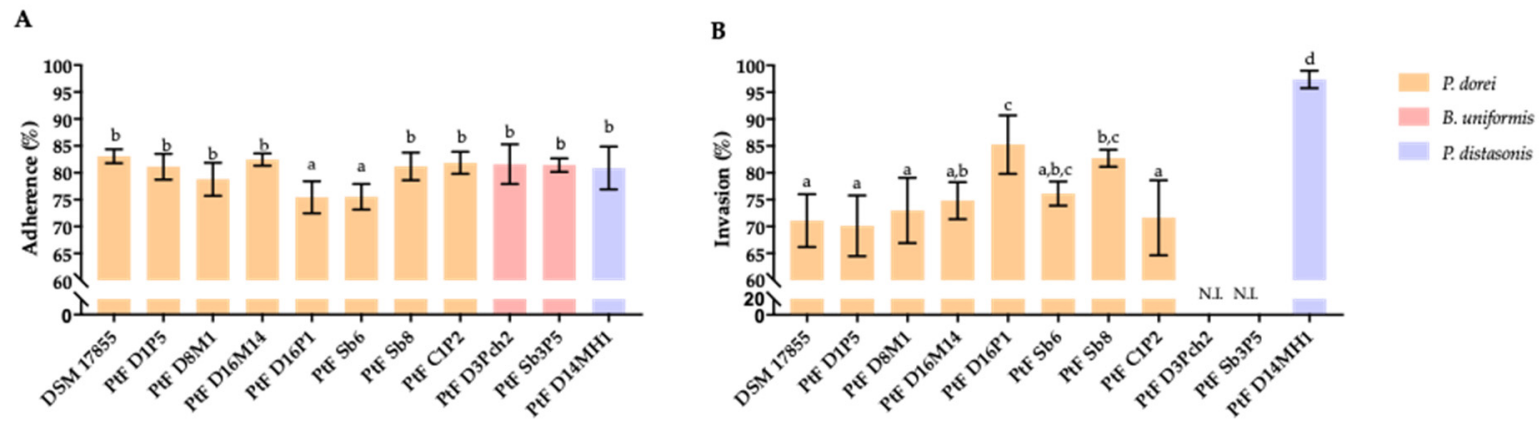

Figure 3. (A) Adherence and (B) invasion ability (\%) of the Bacteroides species tested with HT29-MTX-E12 cell line. Bars with the same lowercase letter are not statistically different $(p>0.05)$. N.I.-non-invasion.

Since T1D children showed a lower number of Lactobacillus sp. by qPCR (Section 3.1) the impact of L. casei DSM 20011 on the invasion ability of P. dorei strains PtF D1P5, PTF $\mathrm{D} 16 \mathrm{P} 1, \mathrm{PtFSb} 8$ and P. distasonis D14MH1 was evaluated. The results are illustrated in Figure 4 . The invasion ability of the $P$. dorei strains was significantly diminished in the presence of $L$. casei $(p<0.05-p<0.01)$, except for the strain P. dorei PtF D16P1, whose invasion ability was not affected by the presence of $L$. casei $(p>0.05)$ (Figure 4 ).

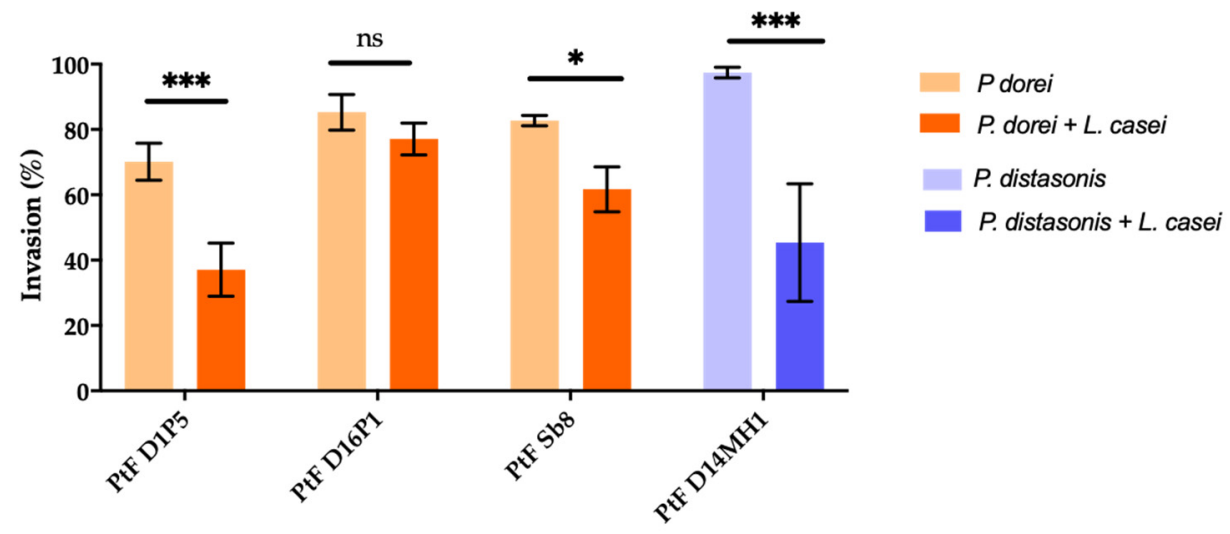

Figure 4. Effect of the presence of Lactobacillus casei DSM 20011 on the invasion ability (\%) of $P$. dorei strains $\left(P\right.$. dorei + L. casei) and P. distasonis $\left(P\right.$. distasonis + L. casei). ${ }^{*} p<0.05 ;{ }^{* *} p<0.001$, ns-not significant.

3.5.1. The Viability of HT29-MTX-E12 Cells Exposed to the Secretome of Bacteroides Species

In order to evaluate the potential impact of the secretome produced by the Bacteroides species on the viability of intestinal epithelial cells, HT-29-MTX-E12 cells were exposed for $24 \mathrm{~h}$ to the culture medium supplemented with different percentages $(10 \%, 25 \%$ and $50 \%)$ of the secretome of Bacteroides cultures. The results are illustrated in Figure 5.

The increase of the secretome concentrations of the reference strain P. dorei DSM 17855, P. dorei PtFD1P5, P. dorei PtFD8M1, and P. dorei PtFC1P2 did not significantly impaired $(p>0.05)$ the viability of HT-29-MTX-E12 cells. In contrast, the exposure of HT-29-MTX-E12 cells to increasing concentrations of the bacterial culture secretome of the strains $P$. dorei PtFD16M14, P. dorei PtFD16P1, P. dorei PtFSb6, P. dorei PtFSb8, B. uniformis PtF D3Pch2, B. 
uniformis PtF Sb5P5, and P. distasonis PtFD14MH1 significantly affected the viability of the intestinal epithelial cells $(p<0.05)$ (Figure 5).
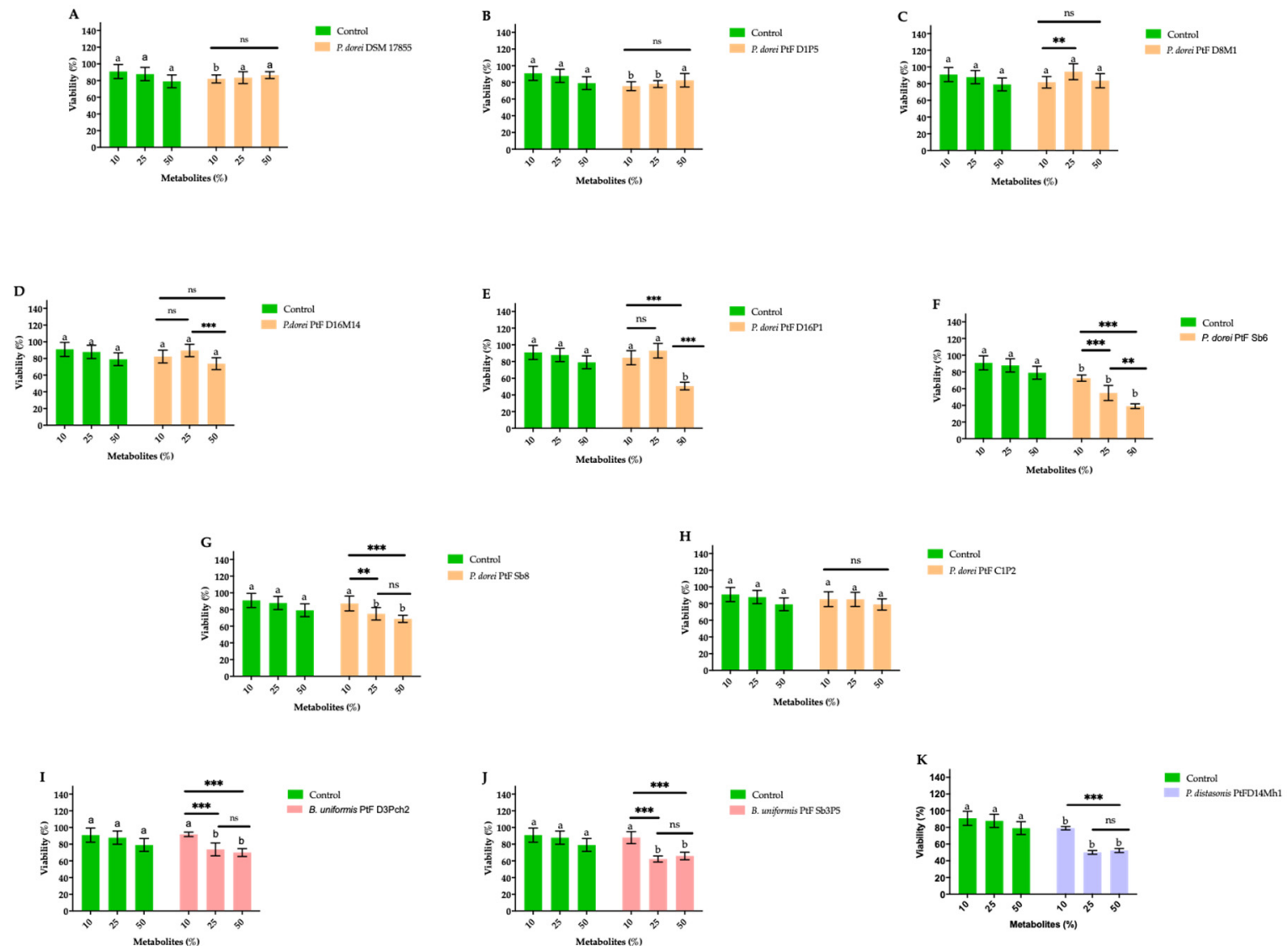

Figure 5. Viability of the HT29-MTX-E12 cells exposed to $10 \%, 25 \%$ and $50 \%$ of the secretome of the culture of P. dorei DSM 17855 (A), P. dorei PtFD1P5 (B), P. dorei PtFD8M1 (C), P. dorei PtFD16M14 (D), P. dorei PtFD16P1 (E), P. dorei PtFSb6 (F), P. dorei PtFSb8 (G), P. dorei PtFC1P2 (H), B. uniformis PtFD3Pch2 (I), B. uniformis PtFSb3P5 (J), P. distasonis PtFD14MH1 (K). The addition of BHI to cell culture at the tested concentrations was used as control. Bars with the same letter are not statistically different when compared to control $(p>0.05)$. Data statistically different for each strain are represented by $* * 0.01$; *** $p<0.001$.

\subsubsection{Integrity of HT29-MTX-E12 Cells after Exposure to Bacteroides Species}

The different strains of P. dorei were able to adhere and invade the HT-29-MTX-E12 cells, except the two tested strains of B. uniformis that were not able to invade. Therefore, the impact of colonization by different bacterial species on tight junction integrity was investigated. The HT-29-MTX-E12 cells were stained with TRICT-phalloidin, a phallotoxin that binds to actin filaments. This binding allows the observation of any damage that has occurred in the network of tight junctions as a result of the injurious action of the Bacteroides species compromising the normal functioning of paracellular communication.

The damage to the tight junctions of HT29-MTX-E12 cells after exposure to P. dorei strains and $P$. distasonis was similar and considerable, in contrast with the cells exposed to $B$. uniformis that were only slightly affected. A representative image of the disturbed tight junctions by the colonization of HT29-MTX-E12 cells with the strain P. dorei PtF D16P1 and B. uniformis PtF D3Pch2 is illustrated in Figure 6. 
A

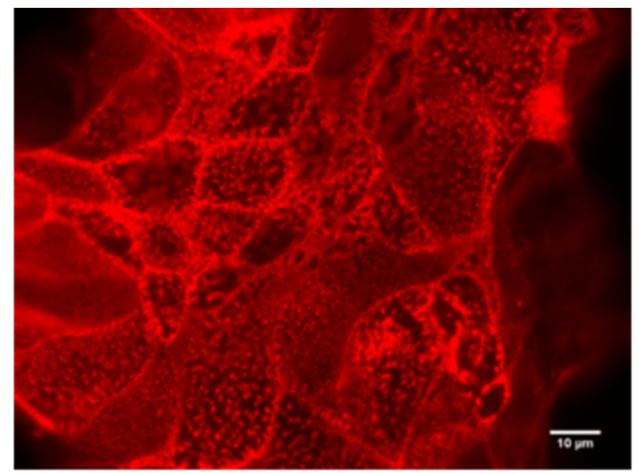

B

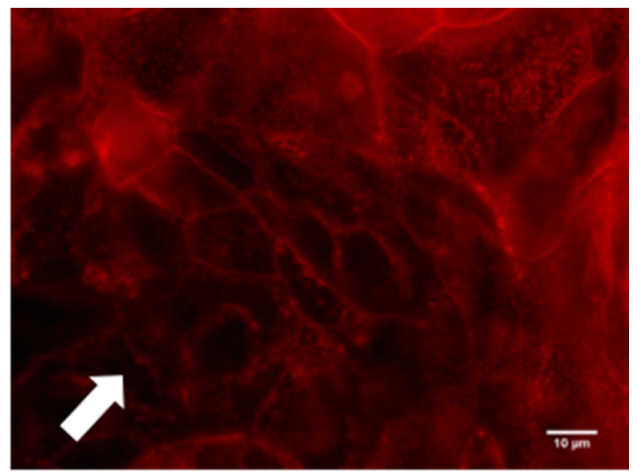

C

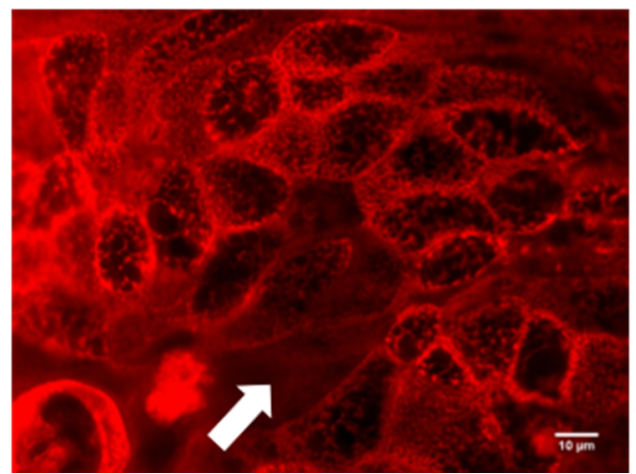

Figure 6. TRITC-phalloidin staining (red) of cell junction's of the cell line HT29-MTXE12. No colonized cells (A), and after $4 \mathrm{~h}$ of colonization with P. dorei PtF D16P1 (B) and B. uniformis PtF D3Pch2 (C). The white arrow indicates evidence of the cell junction's disruptions after the contact with P. dorei PtF D16P1 and B. uniformis PtF D3Pch2 in comparison with the undisrupted cell junctions of the control monolayers.

\subsection{Genomic Features}

Representative circular genome maps for P. dorei, PtFD16P1, B. uniformis PtFD3Pch2 and $P$. distasonis PtFD14MH1 is shown in Figure 7 (the genome map of the remaining seven strains is presented in Figure S3), and their main features are listed in Table 1. The genome size of $P$. dorei strains ranged from $5120 \mathrm{Mb}$ to $5880 \mathrm{Mb}$, and the genome size of P. distasonis PtFD14MH1 (5066 Mb) is similar to the P. dorei strains (Table 1). The genomes of $P$. dorei strains were distributed along 62 to 153 contigs, with the number of contigs for the strain of $P$. distasonis PtFD14MH1 being 81. B. uniformis PtFD3Pch2 showed a similar genome size $(5457 \mathrm{Mb})$ to $P$. dorei strains whereas B. uniformis PtF Sb3P5 showed the lowest genome size, $4393 \mathrm{Mb}$, assembled in 8 and 21 contigs, respectively. The observed GC\% content for the $P$. dorei strains ranged between $41.4 \%$ and $42.2 \%$. In contrast $B$. uniformis strains and $P$. distasonis showed the highest GC \% content, B. uniformis PtFD3Pch2 achieves $44.1 \%$, B. uniformis PtF Sb3P5 46.4\%, and 45\% for P. distasonis PtFD14MH1.

The number of coding genes varied among the $P$. dorei strains, namely the highest number was displayed by the strain PtFD1P5 (5184) and PtFSb6, and the lowest by the strain PtFSb8 (4288) followed by the strain PtFD8M1. The number of coding genes in P. distasonis PtFD14MH1 was similar to those of $P$. dorei strains (4240). The strains of B. uniformis PtFD3Pch2 and PtF Sb5P5 showed 4597 and 3644 coding sequences, respectively.

The distribution of genes by functional categories produced by RAST is summarized in Table S7. Overall, the number of genes in each functional category did not vary markedly between the strains. Some of the categories are highlighted. In the category Phages, Prophages, Transposable elements, and Plasmids, the number of genes in the genomes of P. dorei PtFD8M1, PtFSb8, and P. distasonis PtFD14MH1 are 47, 48, and 33 respectively. However, in this category it is found in the genomes of P. dorei PtFD16P1 and PtFSb8, 4 copies of a thiol-activated cytolysin (LLO) of the subsystem of Listeria Pathogenicity Island LIPI-1. Three copies of this same LLO gene are present in the genomes of strains 
P. dorei PtFD16M14, PtFD8M1, and PtFC1P2. However, no copies of the thiol activated cytolysin were detected in the genome of $P$. dorei PtFSb6. The same four copies number of the thiol-activated cytolysin was found in the genome of B. uniformis PtFD3Pch2. In contrast, in the genome of B. uniformis PtFSb3P5 just 1 copy of the phosphatidylinositol-specific phospholipase $\mathrm{C}$ (PlcA) was identified. In the genome of P. distasonis PtFD14MH1, two copies of this type of the thiol-activated cytolysin were identified. The percentage identity of the identified thiol-activated cytolysins with the listeriolysin $\mathrm{O}$ of L. monocytogenes EGD (lmo0202) is 42.19\% for the strain P. dorei PtF D16P1 (20547_PtFD16P1_01393), 39.98\% for B. uniformis PtFD3Pch2 (20551_D3Pch2_00006) and 48.50\% for P. distasonis D14MH1.

(a)

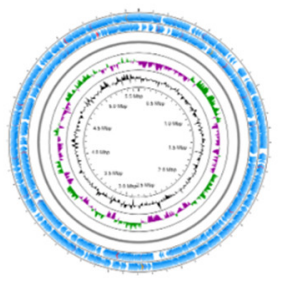

(c)

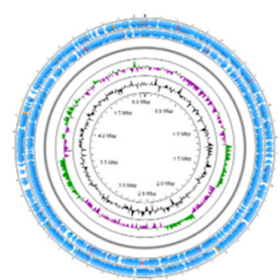

(b)
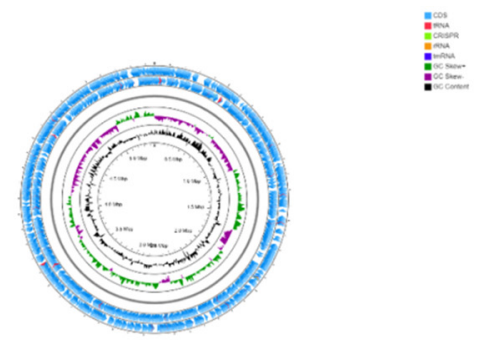

Figure 7. Genome map of P. dorei PtFD16P1 (a), B. uniformis PtFD3Pch2 (b), and P. distasonis PtFD14MH1 (c). The outermost ring depicts the coding sequences in both clockwise and anti-clockwise direction. tRNA is depicted as red arrow heads, rRNA in orange and tmRNA in royal blue. CRISPR repeats regions are coloured in lime green. GC skewing is shown in green and purple, and GC content in black.

Table 1. Genomic features of the analyzed strains.

\begin{tabular}{cccccc}
\hline Strains & Size $\mathbf{( M b )}$ & Contigs & GC $\mathbf{( \% )}$ & Genes & RNAs \\
\hline P. dorei PtFD1P5 & 5880 & 153 & 42.2 & 5184 & 77 \\
P. dorei PtFD8M1 & 5121 & 62 & 41.4 & 4295 & 76 \\
P. dorei PtFD16P1 & 5588 & 101 & 42.1 & 4824 & 75 \\
P. dorei PtFD16M14 & 5592 & 110 & 42.1 & 4835 & 77 \\
P. dorei PtFSb6 & 5849 & 86 & 42.2 & 5164 & 78 \\
P. dorei PtFSb8 & 5120 & 72 & 41.4 & 4288 & 74 \\
P. dorei PtFC1P2 & 5479 & 83 & 41.9 & 4710 & 76 \\
B. uniformis PtFD3Pch2 & 5457 & 8 & 44.1 & 4597 & 59 \\
B. uniformis PtFSb3P5 & 438 & 21 & 46.4 & 3644 & 62 \\
P. distasonis PtFD14MH1 & 5066 & 81 & 45 & 4240 & 85
\end{tabular}

The dendogram obtained from the multilocus sequencing typing is composed of 8 clusters in total (Figure S4). P. dorei strains are distributed in 6 clusters, and the strains of $B$. uniformis and $P$. distasonis are clustered in two independent clusters. The $P$. dorei strains clustered differently with the $P$. dorei strains used as reference. The reference strain $P$. dorei DSM 17855 is the one that is more distant.

The identification of the potential pathogenicity for the human host, performed with PathogenFinder 1.1, revealed the presence of a collagenase precursor (ABR38775) in the P. dorei strains PtF D1P5, PtFD16P1, PtFD16M14, PtFC1P2, and B. uniformis PtFD3Pch2. In P. dorei PtFD16P1 a putative membrane peptidase (ABR39331) was detected. Interestingly, in the genome of $P$. distasonis PtFD14MH1 it was found 4 hemolysin related proteins (ABR 43727,ABR 42607, ABR 44661, and ABR 42685), a metal-dependent hydrolase (ABR42910), 
a putative hydrolase (ABR 42975), a N-acetyl-muramoyl-L-alanine-amidase (ABR 44579), a putative alkaline protease AprF (ABR 41930), a putative aminopeptidase (ABR 42007), and a putative capsule polysaccharide export protein (ABR42909).

Prophage sequences identified by PHASTER are summarized in Table S8. Only incomplete prophages were identified. Among the analyzed bacterial genomes, Flavobacterium phages were the most common putative phage identified, except for P. dorei PtFD1P5, and $P$. distasonis PtFD14MH1. Interestingly, the strain of $P$. dorei PtF C1P2 carry a prophage DNA adenine methylase enzyme (Dam) (PHAGE_Flavob_vB_FspS_morran9_1_NC_048836), and the strains PtFD8M1 and PtFSb8 also show a Flavobacterium phage DNA adenine methylase enzyme (PHAGE_Flavob_vB_FspM_pippi8_1_NC_048830).

B. uniformis PtFD3Pch2 and PtF Sb3P5 show an endolysin from a Rhodococcus phage (PHAGE_Rhodoc_Trina_NC_042040), and a holin from a Bacillus phage (PHAGE_Bacill_ Pookie_NC_027394).

Different sequences of CRISPR were detected in the analyzed genomes (Table S9). Three main CRISPR-Cas types, corresponding essentially to Type IC, Type IIC and Type VIA were identified.

Table 2 summarizes the results of searching for molecular mimicry in the analyzed genomes to identify the presence of insulin B chain insB:9-23 peptide mimics (SHLVEALYL VCGERG) [48], and the two microbial altered peptide ligands (MVWGPDPLYV and RQFGPDWIVA) from the natural preproinsulin (ALWGPDPAAA) [50]. A variant of the insB:9-23 peptide (HLLEALYMTYGE) was identified only in the genome of B. uniformis PtF Sb3P5. In contrast, in all P. dorei genomes and in the genome of the strain B. uniformis PtFD3Pch2, a modified version of the altered peptide MVWGPDPLYV (MVWGPDNFYV) is observed. Interestingly, the strain of B. uniformis PtFD3Pch2 showed another variant of this peptide (MVWSPDPLYV). The P. distasonis PtFD14MH1 showed an altered version of the peptide ligand RQFGPDWIVA.

Table 2. Sequences of the bacterial mimics for human insulin B:9-23 peptide (SHL) and human natural preproinsulin (ALW).

\begin{tabular}{cccc}
\hline Human Insulin/Bacterial Peptide & Sequence & Identity & Reference \\
\hline Human insulin B:9-23 peptide (SHL) & SHLVEALYLVCGERG & Wildtype & {$[48]$} \\
Variant of SHL & HLLEALYMTYGE & B. uniformis PtFSb3P5 & This study \\
Human Natural preproinsulin (ALW) & ALWGPDPAAA & Wildtype & {$[49]$} \\
Variant of ALW & MVWGPDPLY* & Bacteroides fragilis/thetaiotaomicron & {$[49]$} \\
Variant of ALW & MVWGPDNFYV & P. dorei and B. uniformis PtFD3Pch2 & This study \\
Variant of ALW & MVWSPDPLYV & B. uniformis PtFD3Pch2 & This study \\
Variant of ALW & RQFGPDWIVA & Clostridium asparagiforme & {$[49]$} \\
Variant of ALW & RRYGKDWIVA & P. distasonis PtFD14MH1 & This study \\
\hline
\end{tabular}

* Bold letters represent amino acids that are different from the reference preproinsulin-derived sequence.

\section{Discussion}

Nowadays it is clear that geographical location strongly influences the incidence of T1D $[8,10,12,23]$. Even in neighboring countries, the intestinal bacterial patterns observed in children with established T1D does not overlap $[17,18,20]$. This study confirms that Portuguese (Algarve region) children with T1D show similar Bacteroides counts, when compared with control children, both by culture-dependent and qPCR approachs. As it can be anticipated, the slight difference observed between the counts of Bacteroides by the culture-dependent approach and qPCR, is clearly associated with the lower recovery of bacteria in culture medium. Different Bacteroides species were recovered only from T1D children, namely B. graminisolvens, B. cellulosilyticus, B. finegoldii, B. stercoris, B. eggerthii and B. fragilis. Diet can modify the composition of the gut microbiota [54-57]. Bacteroides species are particularly modulated by the accessibility and abundance of dietary fiber [55,58,59], for example arabinoxylans that are naturally found in all major cereal grains increases the abundance of B. cellulosilyticus but not of B. ovatus $[55,60]$. The recommendations in the guidelines for nutritional management in children and adolescents with diabetes, stress the importance of consuming a variety of healthy foods, including fruits, vegetables, 
dairy, whole grains, and legumes [61]. However, the individual consumption of these healthy foods (with a significant load on dietary fiber) will impact the presence of different Bacteroides species in the gut of T1D children [54,55].

Another interesting finding was the higher recovery in culture of $P$. distasonis isolates from the fecal samples of T1D children in comparison with control children. Interestingly, Falony et al. [62] found an increase in Parabacteroides in individuals with low microbiome diversity that exhibited a preference for white, low fiber bread, and predominance of recent amoxicillin treatment. The recent reported molecular mimics of the human insulin B:9-23 peptide in P. distasonis 33B (from the human gut microbiome) [48] is an important warning for hidden autoimmune triggers. The genome of $P$. distasonis PtFD14MH1 did not show the presence of any variant of insB:9-23, however a variant of the Human Natural preproinsulin AWL, that is very similar to a previously described variant in C. asparagiforme, was identified [49]. The role of P. distasonis in host health is still controversial, namely while Huang et al. [48] reported the stimulation by P. distasonis 33B of the human T cell clones specific to insB:9-23 and the colonization of the gut of female NOD mice with $P$. distasonis 33B stimulate T1D onset, Wang et al. [63] reported that the treatment of obese mice with live $P$. distasonis resulted in an increase in the concentration of succinate in the jejunum, together with the activation of intestinal gluconeogenesis, diminishing food intake, and amelioration of glucose homeostasis. Other beneficial features of $P$. distasonis, is its ability to mitigate obesity-driven colorectal tumorigenesis and attenuate intestinal inflammation $[64,65]$. Altogether these findings reveal the controversial nature of the role of $P$. distasonis on gut inflammatory diseases. Nevertheless, it is reasonable to anticipate that such contrasting behavior may be highly strain-specific, and obviously the genetic background of the host is also a decisive factor. The reason for the higher recovery of $P$. distasonis isolates from T1D in comparison to control samples, and how their molecular mimicry can contribute to the development of T1D, requires additional research.

Repetitive sequence-based PCR banding patterns obtained from both BOX and ERIC primers, grouped the different Bacteroides species, but no discrimination by origin (cases and control) was observed. The rep-PCR method is directed to DNA sequences that are dispersed in the genome of the microorganism, and have been used to find differences in the bacterial genomes that will help with the discrimination between bacterial isolates, including the different Bacteroides species [30]. As the majority of bacteria, Bacteroides carry mobile genetic elements, such as plasmids, conjugative and mobilizable transposons [66]. In the host, these mobilizable genetic elements can enable the exchange of external DNA from the gut members, which can result in different rep-PCR banding profiles. The lack of differentiation of the Bacteroides species according to their sampling origin, lead us to assume that the host condition (T1D or healthy) has no impact on the coevolution of these Bacteroides species.

T1D children showed a similar ratio of Firmicutes to Bacteroidetes when compared to control children, but a significant increase in Proteobacteria and Prevotella sp., which is in contrast with the findings of Murri et al. [17] that observed a significantly higher ratio of Firmicutes to Bacteroidetes, and higher numbers of Prevotella sp. in healthy children with no significant differences in the numbers of Proteobacteria. The deviation of data regarding these bacterial groups can be justified by differences in geographical location factors $[10,12,23]$. However, the number of Lactobacillus sp. was impaired in T1D children, which is in line with the results reported by Murri et al. [17] in children with established T1D, and with the findings described by Biassoni et al. [67] in children at T1D onset, as well as with findings using rodent models, namely in bio-breeding diabetes prone mice [68] and streptozotocin T1D rat model $[69,70]$.

All tested P. dorei and P. vulgatus strains were able to grow in the presence of porcine gastric mucin $(1 \%[w / v])$, but in a limited way when compared with their growth in the presence of glucose $(0.5 \%, w / v)$ or in complex medium $(\mathrm{BHI}+\mathrm{H})$. The ability to break mucin, evidenced by amidoblack staining, was confirmed for all tested strains. Bacteroides species are recognized by their ability to degrade dietary fiber and host intestinal 
mucin $[57,71]$. Taking this into account, and knowing that hyperglycemia affects the intestinal mucus layer allowing a diminished microbiota-epithelial distance [72], P. dorei, $P$. vulgatus and other Bacteroidetes members in T1D gut may contribute to a disturbed mucus layer, therefore facilitating bacterial infiltration.

The ability to adhere to HT-29-MTX cells was similarly observed for all tested strains of P. dorei, B. uniformis and the strain of P. distasonis PtFD14MH1. The ability of different Bacteroides species and $P$. distasonis isolates, collected from a healthy donor for fecal microbiota transplantation (FMT), to adhere to the intestinal epithelial cells HT-29 and Caco-2 (non-mucus secreting cell) was recently reported [65]. The authors observed that the three isolates of $B$. caccae, and among the six isolates of $P$. distasonis only one was able to adhere to HT-29 and Caco-2 cells [65]. Our contrasting findings can be explained by the differences between the strains and the cell line tested (a mucus secreting cell).

In this study it was observed that the ability to invade HT-29-MTX cells was different between species and strains, where the highest ability to invade was achieved by $P$. distasonis PtFD14MH1 followed by P. dorei strains PtF D16P1 and PtF Sb8. The B. uniformis strains were not able to invade HT-29-MTX cells. A severe damage to the tight junctions of HT29-MTX cells after exposure to $P$. dorei strains and $P$. distasonis was observed, in contrast with the B. uniformis strains that cause a minor disturbance. To the best of our knowledge, this is the first study that reports the ability of Bacteroides species and Parabacteroides to invade mucus secreting cells. Different number of copies of the thiol-activated cytolysin (Listeriolysin O, LLO) of the subsystem of Listeria Pathogenicity Island LIPI-1 were identified in the genome of the $P$. dorei strains, B. uniformis and P. distasonis PtFD14MH1, except in the strain P. dorei PtFSb6. It is known that LLO, a pore-forming toxin is able to exert lytic activity in different types of cells, including the intestinal epithelial cell Caco-2 [73-75]. The presence of pore-forming toxins in the genome of $P$. dorei was recently described in the strain P. dorei CL03T12C01, including a Hly-III homologue [76]. The transepithelial electrical resistance (TEER) of Caco-2 cells decreases very rapidly (30s) after exposure to LLO at $1 \mu \mathrm{M}$ [75]. A similar impairment of TEER values in the HT-29/B6 human colon cells was previously reported [77]. The decline in TEER values reported by Cajnko et al. [75] was associated to pore formation and to the disturbance of the tight junction protein claudin-1. Several bacteria, namely group A Streptococcus and S. agalactiae are able to increase intestinal permeability by disrupting tight junctions, which allows their paracellular passage [78]. The work of Vieira et al. [79] evidenced the ability of Enterococcus gallinarum to break the gut barrier being translocated to the liver, spleen and mesenteric lymph nodes, leading to the development of autoimmunity. The extent to which the thiol-activated cytolysins found in our strains contributes to the invasion of intestinal epithelial cells and disruption of tight junctions is one of our current research lines.

It was reported that the $1 \mathrm{E} 6$ human $\mathrm{CD} 8+\mathrm{T}$ cell clone, which intermediates the damage of $\beta$ cells by recognizing the major HLA-A*0201-restricted, preproinsulin signal peptide (ALWGPDPAAA $15-24, \mathrm{AWL}$ ) is able to recognize a massive number of different peptides [80]. Moreover, insulitic lesions in T1D patients show a significant occupancy with CD8+ T cells that recognize HLA-A*0201-ALWGPDPAAA [81]. The variants of the preproinsulin signal peptide AWL with sequence MVWGPDPLYV and RQFGPDWIVA were identified in B. fragilis/thetaiotaomicron and C. asparagiforme, and this latter variant was able to activate the 1E6 T cell with higher potency than the ALW sequence highlighting the potential development of autoimmunity by the T- cell cross-reactivity with bacterial derived peptides [49]. The results of the molecular mimicry search in the present study showed the presence of variants of the human natural preproinsulin ALW in the strains of $P$. dorei, B. uniformis and P. distasonis. It is important to highlight that P. distasonis PtFD14MH1 carries a variant similar to the one found in C. asparagiforme (RRYGKDWIVA); in contrast the strains of $P$. dorei and B. uniformis carry a variant similar to the one found in $B$. fragilis / thetaiotaomicron (MVWGPDNFYV). Intriguing, only the strain of B. uniformis PtFSb3P5 showed a variant of the human insulin B:9-23 peptide (HLLEALYMTYGE, SHL). The reported variant of the SHL in P. distasonis 33B (RILVELLYLVCSEYL) is able to promote an 
immune response to the endogenous insB:9-23, and the exposure of female NOD mice to $P$. distasonis mimic peptide during the gut microbiota maturing precipitated the onset of T1D [49]. Our findings, together with the others discussed, empower the hypothesis that common gut bacteria may injure the gut integrity, allowing for bacterial dissemination and eliciting an immune response that, in susceptible hosts, may trigger autoimmunity [82].

Several studies have reported the beneficial role of different Lactobacillus species on delaying the development of T1D or reducing its complications, using mice models [83-86]. In the current study the fecal samples of T1D children showed lower numbers of Lactobacillus spp. in comparison with control children, and such findings were also observed in fecal samples of Spanish T1D children [17]. Interestingly, we observed that the invasive ability of P. dorei and P. distasonis PtFD14MH1, using HT-29-MTX cells was impaired in the presence of L. casei DSM 20011, except for the strain P. dorei PtFD16P1. Probiotics (non-pathogenic microorganisms that are capable of protecting and promoting the health status of the host, such as Lactobacillus spp.) can act against pathogens through different mechanisms, namely by producing antibacterial substances like bacteriocins that prevent their replication, competition for scarce nutrients in the host, anti-adhesive and anti-invasive action, and competitive exclusion by obstructing the adherence of pathogens to host cell binding sites $[87,88]$. One of the great benefits that $L$. casei strains can possibly exert against the invasion ability of our tested strains of $P$. dorei and P. distasonis is its known capacity to promote the abundance of tight junction proteins and mucin $[87,89]$. The inability of L. casei DSM 20011 to inhibit the invasion of strain P. dorei PtFD16P1 requires further investigation.

The viability of HT-29-MTX-E12 cells was impaired by the exposure to the secretome of several P. dorei strains, the strains of B. uniformis and the P. distasonis PtF D14MH1. Recently the impact of the secretome of 157 gut bacteria on the growth rates of 5 colorectal cancer cell lines (CRC), including HT-29 was evaluated [90], and among these 157 gut bacteria, 12 belonged to the genus Bacteroides comprising the species P. dorei, B. eggerthii, B. fragilis, B. massiliensis, and B. ovatus. The effect of the Bacteroides species on cell growth of HT-29 cell line varied from an enhancement to a decline [90]. The negative impact of Bacteroides species on the growth of the CRC lines was attributed to the action of lipoproteins at the bacterial surface that have a high affinity for cobalamin, confiscating it from the culture medium, making this crucial vitamin inaccessible to the human cells, hence impairing their growth $[90,91]$. We can anticipate that besides the role of lipoproteins, other components of the secretome of our strains may be involved in the impairment of the viability of HT-29-MTX-E12 cells, namely the bacterial protease arsenal, which remains to be studied.

\section{Conclusions}

The recovery of Bacteroides species from the fecal samples of T1D and control children allowed us to expand our knowledge about their potential contribution to elicit adverse effects in susceptible hosts such as T1D children. The reason for the higher recovery of $P$. distasonis from T1D fecal samples demands additional investigation. It is noteworthy the observed invasion ability and capacity of some $P$. dorei strains and $P$. distasonis PtFD14MH1 to damage the tight junctions of the intestinal epithelial cells. The depletion of Lactobacillus spp. on the gut microbiota of T1D children was noticed, and its ability to inhibit the invasion ability of the tested strains was remarkable. These data together with other previous reports, evidence the continued efforts that have to be made to find the necessary balance between the gut microbiota of T1D children.

Our data also highlights the need to explore the contribution of the molecular mimicry, identified in the tested strains, to the development of autoimmunity, and offer new opportunities to find tools that can prevent and treat this disease that has such high prevalence and causes severe comorbities in medium to long term. 
Supplementary Materials: The following are available online at https: / www.mdpi.com/article/ 10.3390/microorganisms9071436/s1, Figure S1: Growth in Brain Heart Infusion (BHI), Defined medium with glucose (DM GLU) and Defined medium supplemented with porcine gastric mucin $(1 \%, w / v)(D M M U C I N)$, Figure S2: Cluster analysis by BOX rep-PCR (a) and ERIC rep-PCR (b) of Bacteroides species, Phocaeicola dorei and Parabacteroides distasonis isolated from T1D and Control children, Figure S3: Genome map of P. dorei PtFD1P5 (a), P. dorei PtF D8M1 (b), P. dorei PtF D16M14 (c) , P. dorei PtF Sb8 (d), P. dorei PtF Sb6 (e) P.dorei PtFC1P2 (f) and B. uniformis PtFSb3P5 (g), Figure S4: Phylogenetic relationships of the whole genome sequenced strains of $P$. dorei, B. uniformis and $P$. distasonis based on 30 genes, Table S1: Primers used in qPCR, Table S2: qPCR reaction conditions used for the tested bacterial groups, Table S3: Genes selected for MLSA, Table S4: Antibiotic susceptibility, Table S5: Minimum Inhibitory Concentration of Imipinem by $\varepsilon$ test, Table S6: Identification of resistance genes in the genomes of $P$. dorei, B. uniformis and $P$. distasonis strains using the CARD/RGI, Table S7: SEED subsystem category distribution of the genomes of $P$. dorei, B. uniformis, and $P$. distasonis strains according to RAST annotation, Table S8: Identification of prophage sequences using PHASTER, Table S9: Identification of sequences of CRISPR/Cas in the analyzed genomes using CRISPRCasFinder.

Author Contributions: Conceptualization of the project and experiment design, M.L.F.; provision of fecal samples, M.C.; investigation, J.M., I.M., P.S., I.D. and Y.C.; writing-original manuscript draft, all authors contributed; funding acquisition, M.L.F. All authors have read and agreed to the published version of the manuscript.

Funding: This research was partially funded by Maratona da Saúde and Fundação para a Ciência e Tecnologia through the project UID/BIM/04773/2019 CBMR.

Institutional Review Board Statement: The study was conducted according to the guidelines of the Declaration of Helsinki, and approved by the Administrative Council of the Algarve University Hospital Center through the dispatch of 10/10/2016.

Informed Consent Statement: Informed consent was obtained from all parents of the participating children.

Acknowledgments: The authors are grateful to Eric Triplett and Jordan Russel from the University of Florida, Department of Microbiology and Cell Science for guidance on Bacteroides isolation. The authors also are thankful for the imaging analysis using the equipment available at Light Microscopy Unit of ABC-UAlg that was partially supported by National Portuguese funding PPBI-POCI-01-0145FEDER-22122.

Conflicts of Interest: The authors declare no conflict of interest.

\section{References}

1. Jerram, S.T.; Leslie, R.D. The genetic architecture of type 1 diabetes. Genes 2017, 8, 209. [CrossRef]

2. Knip, M.; Veijola, R.; Virtanen, S.M.; Hyoty, H.; Vaarala, O.; Akerblom, H.K. Environmental Triggers and determinants of Type 1 Diabetes. Diabetes 2005, 54, S125-S136. [CrossRef]

3. Davis-Richardson, A.G.; Triplett, E.W. A model for the role of gut bacteria in the development of autoimmunity for type 1 diabetes. Diabetologia 2015, 58, 1386-1393. [CrossRef]

4. Vatanen, T.; Kostic, A.D.; D’Hennezel, E.; Siljander, H.; Franzosa, E.A.; Yassour, M.; Kolde, R.; Vlamakis, H.; Arthur, T.D.; Hämäläinen, A.M.; et al. Variation in Microbiome LPS Immunogenicity Contributes to Autoimmunity in Humans. Cell 2016, 165, 842-853. [CrossRef] [PubMed]

5. Xu, J.; Huang, G.; Nagy, T.; Guo, T.L. Bisphenol A alteration of type 1 diabetes in non-obese diabetic (NOD) female mice is dependent on window of exposure. Arch. Toxicol. 2019. [CrossRef] [PubMed]

6. Beyerlein, A.; Donnachie, E.; Ziegler, A.G. Infections in Early Life and Development of Type 1 Diabetes. JAMA 2016, 315, 1899-1901. [CrossRef] [PubMed]

7. Alkanani, A.K.; Hara, N.; Gottlieb, P.A.; Ir, D.; Robertson, C.E.; Wagner, B.D.; Frank, D.N.; Zipris, D. Alterations in intestinal microbiota correlate with susceptibility to type 1 diabetes. Diabetes 2015, 64, 3510-3520. [CrossRef] [PubMed]

8. Davis-Richardson, A.G.; Ardissone, A.N.; Dias, R.; Simell, V.; Leonard, M.T.; Kemppainen, K.M.; Drew, J.C.; Schatz, D.; Atkinson, M.A.; Kolaczkowski, B.; et al. Bacteroides dorei dominates gut microbiome prior to autoimmunity in Finnish children at high risk for type 1 diabetes. Front. Microbiol. 2014, 10, 678. [CrossRef] [PubMed]

9. De Goffau, M.C.; Fuentes, S.; Van Den Bogert, B.; Honkanen, H.; De Vos, W.M.; Welling, G.W.; Hyöty, H.; Harmsen, H.J.M. Aberrant gut microbiota composition at the onset of type 1 diabetes in young children. Diabetologia 2014, 57, 1569-1577. [CrossRef] 
10. Endesfelder, D.; Castell, W.Z.; Ardissone, A.; Davis-Richardson, A.G.; Achenbach, P.; Hagen, M.; Pflueger, M.; Gano, K.A.; Fagen, J.R.; Drew, J.C.; et al. Compromised gut microbiota networks in children with anti-islet cell autoimmunity. Diabetes 2014, 63, 2006-2014. [CrossRef]

11. Giongo, A.; Gano, K.A.; Crabb, D.B.; Mukherjee, N.; Novelo, L.L.; Casella, G.; Drew, J.C.; Ilonen, J.; Knip, M.; Hyöty, H.; et al. Toward defining the autoimmune microbiome for type 1 diabetes. ISME J. 2011, 5, 82-91. [CrossRef]

12. Kemppainen, K.M.; Ardissone, A.N.; Davis-Richardson, A.G.; Fagen, J.R.; Gano, K.A.; León-Novelo, L.G.; Vehik, K.; Casella, G.; Simell, O.; Ziegler, A.G.; et al. Early childhood gut microbiomes show strong geographic differences among subjects at high risk for type 1 diabetes. Diabetes Care 2015, 38, 329-332. [CrossRef]

13. Kostic, A.D.; Gevers, D.; Siljander, H.; Vatanen, T.; Hyötyläinen, T.; Hämäläinen, A.M.; Peet, A.; Tillmann, V.; Pöhö, P.; Mattila, I.; et al. The dynamics of the human infant gut microbiome in development and in progression toward type 1 diabetes. Cell Host Microbe 2015, 17, 260-273. [CrossRef] [PubMed]

14. Mejía-León, M.E.; Petrosino, J.F.; Ajami, N.J.; Domínguez-Bello, M.G.; De La Barca, A.M.C. Fecal microbiota imbalance in Mexican children with type 1 diabetes. Sci. Rep. 2014, 4, 3814. [CrossRef]

15. Soyucen, E.; Gulcan, A.; Aktuglu-Zeybek, A.C.; Onal, H.; Kiykim, E.; Aydin, A. Differences in the gut microbiota of healthy children and those with type 1 diabetes. Pediatr. Int. 2014, 56, 336-343. [CrossRef]

16. Vatanen, T.; Franzosa, E.A.; Schwager, R.; Tripathi, S.; Arthur, T.D.; Vehik, K.; Lernmark, Å.; Hagopian, W.A.; Rewers, M.J.; She, J.X.; et al. The human gut microbiome in early-onset type 1 diabetes from the TEDDY study. Nature 2018, 562, 589-594. [CrossRef]

17. Murri, M.; Leiva, I.; Gomez-Zumaquero, J.M.; Tinahones, F.J.; Cardona, F.; Soriguer, F.; Queipo-Ortuño, M.I. Gut microbiota in children with type 1 diabetes differs from that in healthy children: A case-control study. BMC Med. 2013, 11, 46. [CrossRef]

18. Pinto, E.; Anselmo, M.; Calha, M.; Bottrill, A.; Duarte, I.; Andrew, P.W.; Faleiro, M.L. The intestinal proteome of diabetic and control children is enriched with different microbial and host proteins. Microbiology 2017, 163. [CrossRef]

19. Qi, C.J.; Zhang, Q.; Yu, M.; Xu, J.P.; Zheng, J.; Wang, T.; Xiao, X.H. Imbalance of fecal microbiota at newly diagnosed type 1 diabetes in Chinese children. Chin. Med. J. 2016, 129, 1298-1304. [CrossRef]

20. Leiva-Gea, I.; Sánchez-Alcoholado, L.; Martín-Tejedor, B.; Castellano-Castillo, D.; Moreno-Indias, I.; Urda-Cardona, A.; Tinahones, F.J.; Fernández-García, J.C.; Queipo-Ortuño, M.I. Gut microbiota differs in composition and functionality between children with type 1 diabetes and MODY2 and healthy control subjects: A case-control study. Diabetes Care 2018, 41, 2385-2395. [CrossRef]

21. De Goffau, M.C.; Luopajärvi, K.; Knip, M.; Ilonen, J.; Ruohtula, T.; Härkönen, T.; Orivuori, L.; Hakala, S.; Welling, G.W.; Harmsen, H.J.; et al. Fecal microbiota composition differs between children with $\beta$-cell autoimmunity and those without. Diabetes 2013, 62, 1238-1244. [CrossRef]

22. García-López, M.; Meier-Kolthoff, J.P.; Tindall, B.J.; Gronow, S.; Woyke, T.; Kyrpides, N.C.; Hahnke, R.L.; Göker, M. Analysis of 1,000 Type-Strain Genomes Improves Taxonomic Classification of Bacteroidetes. Front. Microbiol. 2019, 10. [CrossRef]

23. Cinek, O.; Kramna, L.; Lin, J.; Oikarinen, S.; Kolarova, K.; Ilonen, J.; Simell, O.; Veijola, R.; Autio, R.; Hyöty, H. Imbalance of bacteriome profiles within the Finnish Diabetes Prediction and Prevention study: Parallel use of 16S profiling and virome sequencing in stool samples from children with islet autoimmunity and matched controls. Pediatr. Diabetes 2017, 18, 588-598. [CrossRef]

24. Bacic, M.K.; Smith, C.J. Laboratory Maintenance and Cultivation of Bacteroides Species. Curr. Protoc. Microbiol. 2008, 9, 13C.1.1-13C.1.21. [CrossRef]

25. Pitcher, D.G.; Saunders, N.A.; Owen, R.J. Rapid extraction of bacterial genomic DNA with guanidium thiocyanate. Lett. Appl. Microbiol. 1989, 8, 151-156. [CrossRef]

26. Bakir, M.A.; Sakamoto, M.; Kitahara, M.; Matsumoto, M.; Benno, Y. Bacteroides dorei sp. nov., isolated from human faeces. Int. J. Syst. Evol. Microbiol. 2006, 56, 1639-1643. [CrossRef]

27. Altschul, S.F.; Gish, W.; Miller, W.; Myers, E.W.; Lipman, D.J. Basic local alignment search tool. J. Mol. Biol. 1990, 215, 403-410. [CrossRef]

28. Cole, J.R.; Wang, Q.; Fish, J.A.; Chai, B.; McGarrell, D.M.; Sun, Y.; Brown, C.T.; Porras-Alfaro, A.; Kuske, C.R.; Tiedje, J.M. Ribosomal Database Project: Data and tools for high throughput rRNA analysis. Nucleic Acids Res. 2014, 42, 633-642. [CrossRef]

29. Versalovic, J.; Koeuth, T.; Lupski, J.R. Distribution of repetitive DNA sequences in eubacteria and application to fingerprinting of bacterial genomes. Nucleic Acids Res. 1991, 19, 6823-6831. [CrossRef]

30. Atherly, T.; Ziemer, C.J. Bacteroides isolated from four mammalian hosts lack host-specific 16S rRNA gene phylogeny and carbon and nitrogen utilization patterns. Microbiologyopen 2014, 3, 225-238. [CrossRef]

31. Ho, P.L.; Yau, C.Y.; Ho, L.Y.; Lai, E.L.Y.; Liu, M.C.J.; Tse, C.W.S.; Chow, K.H. Antimicrobial susceptibility of Bacteroides fragilis group organisms in Hong Kong by the tentative EUCAST disc diffusion method. Anaerobe 2017, 47, 51-56. [CrossRef]

32. Nagy, E.; Justesen, U.S.; Eitel, Z.; Urbán, E. Development of EUCAST disk diffusion method for susceptibility testing of the Bacteroides fragilis group isolates. Anaerobe 2015, 31, 65-71. [CrossRef]

33. European Committee on Antimicrobial Susceptibility Testing. Breakpoint Tables for Interpretation of MICs and Zone Diameters. Available online: http:/ / www.eucast.org (accessed on 4 June 2021).

34. Yesilkaya, H.; Manco, S.; Kadioglu, A.; Terra, V.S.; Andrew, P.W. The ability to utilize mucin affects the regulation of virulence gene expression in Streptococcus pneumoniae. FEMS Microbiol. Lett. 2008, 278, 231-235. [CrossRef] 
35. Bolger, A.M.; Lohse, M.; Usadel, B. Trimmomatic: A flexible trimmer for Illumina sequence data. Bioinformatics 2014, 30, 2114-2120. [CrossRef]

36. Bankevich, A.; Nurk, S.; Antipov, D.; Gurevich, A.A.; Dvorkin, M.; Kulikov, A.S.; Lesin, V.M.; Nikolenko, S.I.; Pham, S.; Prjibelski, A.D.; et al. SPAdes: A new genome assembly algorithm and its applications to single-cell sequencing. J. Comput. Biol. 2012, 19, 455-477. [CrossRef]

37. Seemann, T. Prokka: Rapid prokaryotic genome annotation. Bioinformatics 2014, 30, 2068-2069. [CrossRef]

38. Silva, G.G.Z.; Dutilh, B.E.; Matthews, T.D.; Elkins, K.; Schmieder, R.; Dinsdale, E.A.; Edwards, R.A. Combining de novo and reference-guided assembly with scaffold_builder. Source Code Biol. Med. 2013, 8, 23. [CrossRef]

39. Petkau, A.; Stuart-Edwards, M.; Stothard, P.; van Domselaar, G. Interactive microbial genome visualization with GView. Bioinformatics 2010, 26, 3125-3126. [CrossRef]

40. Chun, J.; Oren, A.; Ventosa, A.; Christensen, H.; Arahal, D.R.; da Costa, M.S.; Rooney, A.P.; Yi, H.; Xu, X.W.; De Meyer, S.; et al. Proposed minimal standards for the use of genome data for the taxonomy of prokaryotes. Int. J. Syst. Evol. Microbiol. 2018, 68, 461-466. [CrossRef]

41. Munoz, R.; Rosselló-Móra, R.; Amann, R. Revised phylogeny of Bacteroidetes and proposal of sixteen new taxa and two new combinations including Rhodothermaeota phyl. nov. Syst. Appl. Microbiol. 2016, 39, 491-492. [CrossRef] [PubMed]

42. Sievers, F.; Wilm, A.; Dineen, D.; Gibson, T.J.; Karplus, K.; Li, W.; Lopez, R.; McWilliam, H.; Remmert, M.; Söding, J.; et al. Fast, scalable generation of high-quality protein multiple sequence alignments using Clustal Omega. Mol. Syst. Biol. 2011, 7. [CrossRef]

43. Kumar, S.; Stecher, G.; Li, M.; Knyaz, C.; Tamura, K. MEGA X: Molecular evolutionary genetics analysis across computing platforms. Mol. Biol. Evol. 2018, 35, 1547-1549. [CrossRef]

44. Arndt, D.; Grant, J.R.; Marcu, A.; Sajed, T.; Pon, A.; Liang, Y.; Wishart, D.S. PHASTER: A better, faster version of the PHAST phage search tool. Nucleic Acids Res. 2016, 44, W16-W21. [CrossRef]

45. Couvin, D.; Bernheim, A.; Toffano-Nioche, C.; Touchon, M.; Michalik, J.; Néron, B.; Rocha, E.P.C.; Vergnaud, G.; Gautheret, D.; Pourcel, C. CRISPRCasFinder, an update of CRISRFinder, includes a portable version, enhanced performance and integrates search for Cas proteins. Nucleic Acids Res. 2018, 46, W246-W251. [CrossRef] [PubMed]

46. Alcock, B.P.; Raphenya, A.R.; Lau, T.T.Y.; Tsang, K.K.; Bouchard, M.; Edalatmand, A.; Huynh, W.; Nguyen, A.L.V.; Cheng, A.A.; Liu, S.; et al. CARD 2020: Antibiotic resistome surveillance with the comprehensive antibiotic resistance database. Nucleic Acids Res. 2020, 48, D517-D525. [CrossRef]

47. Cosentino, S.; Voldby Larsen, M.; Møller Aarestrup, F.; Lund, O. PathogenFinder-Distinguishing Friend from Foe Using Bacterial Whole Genome Sequence Data. PLoS ONE 2013, 8. [CrossRef]

48. Huang, Q.; Chow, I.-T.; Brady, C.; Raisingani, A.; Li, D.; Ostrov, D.A.; Atkinson, M.A.; Kwok, W.K.; Kahn, C.; Ronald, A.E. Parabacteroides distasonis enhances Type 1 Diabetes autoimmunity via molecular mimicry. bioRxiv 2020, 9-23. [CrossRef]

49. Cole, D.K.; Bulek, A.M.; Dolton, G.; Schauenberg, A.J.; Szomolay, B.; Rittase, W.; Trimby, A.; Jothikumar, P.; Fuller, A.; Skowera, A.; et al. Hotspot autoimmune T cell receptor binding underlies pathogen and insulin peptide cross-reactivity. J. Clin. Investig. 2016, 126, 2191-2204. [CrossRef]

50. Gagnon, M.; Zihler Berner, A.; Chervet, N.; Chassard, C.; Lacroix, C. Comparison of the Caco-2, HT-29 and the mucus-secreting HT29-MTX intestinal cell models to investigate Salmonella adhesion and invasion. J. Microbiol. Methods 2013, 94, 274-279. [CrossRef]

51. Veloo, A.C.M.; Baas, W.H.; Haan, F.J.; Coco, J.; Rossen, J.W. Prevalence of antimicrobial resistance genes in Bacteroides spp. and Prevotella spp. Dutch clinical isolates. Clin. Microbiol. Infect. 2019, 25, 1156.e9-1156.e13. [CrossRef]

52. Madinier, I.; Fosse, T.; Giudicelli, J.; Labia, R. Cloning and biochemical characterization of a class A $\beta$-lactamase from Prevotella intermedia. Antimicrob. Agents Chemother. 2001, 45, 2386-2389. [CrossRef]

53. Stentz, R.; Horn, N.; Cross, K.; Salt, L.; Brearley, C.; Livermore, D.M.; Carding, S.R. Cephalosporinases associated with outer membrane vesicles released by Bacteroides spp. protect gut pathogens and commensals against $\beta$-lactam antibiotics. J. Antimicrob. Chemother. 2015, 70, 701-709. [CrossRef] [PubMed]

54. Townsend, G.E.; Han, W.; Schwalm, N.D.; Raghavan, V.; Barry, N.A.; Goodman, A.L.; Groisman, E.A. Dietary sugar silences a colonization factor in a mammalian gut symbiont. Proc. Natl. Acad. Sci. USA 2019, 116, 233-238. [CrossRef]

55. Wu, M.; McNulty, N.P.; Rodionov, D.A.; Khoroshkin, M.S.; Griffin, N.W.; Cheng, J.; Latreille, P.; Kerstetter, R.A.; Terrapon, N.; Henrissat, B.; et al. Genetic determinants of in vivo fitness and diet responsiveness in multiple human gut Bacteroides. Science 2015, 350. [CrossRef]

56. Sawicki, C.M.; Livingston, K.A.; Obin, M.; Roberts, S.B.; Chung, M.; McKeown, N.M. Dietary fiber and the human gut microbiota: Application of evidence mapping methodology. Nutrients 2017, 9, 125. [CrossRef]

57. Gamage, H.K.A.H.; Chong, R.W.W.; Bucio-Noble, D.; Kautto, L.; Hardikar, A.A.; Ball, M.S.; Molloy, M.P.; Packer, N.H.; Paulsen, I.T. Changes in dietary fiber intake in mice reveal associations between colonic mucin O-glycosylation and specific gut bacteria. Gut Microbes 2020, 12. [CrossRef]

58. Patnode, M.L.; Beller, Z.W.; Han, N.D.; Cheng, J.; Peters, S.L.; Terrapon, N.; Henrissat, B.; Le Gall, S.; Saulnier, L.; Hayashi, D.K.; et al. Interspecies Competition Impacts Targeted Manipulation of Human Gut Bacteria by Fiber-Derived Glycans. Cell 2019, 179, 59-73.e13. [CrossRef] [PubMed] 
59. Sonnenburg, E.D.; Zheng, H.; Joglekar, P.; Higginbottom, S.K.; Firbank, S.J.; Bolam, D.N.; Sonnenburg, J.L. Specificity of polysaccharide use in intestinal bacteroides species determines diet-induced microbiota alterations. Cell 2010, 141, 1241-1252. [CrossRef] [PubMed]

60. McNulty, N.P.; Wu, M.; Erickson, A.R.; Pan, C.; Erickson, B.K.; Martens, E.C.; Pudlo, N.A.; Muegge, B.D.; Henrissat, B.; Hettich, R.L.; et al. Effects of Diet on Resource Utilization by a Model Human Gut Microbiota Containing Bacteroides cellulosilyticus WH2, a Symbiont with an Extensive Glycobiome. PLoS Biol. 2013, 11. [CrossRef] [PubMed]

61. Smart, C.E.; Annan, F.; Higgins, L.A.; Jelleryd, E.; Lopez, M.; Acerini, C.L. ISPAD Clinical Practice Consensus Guidelines 2018: Nutritional management in children and adolescents with diabetes. Pediatr. Diabetes 2018, 19, 136-154. [CrossRef]

62. Falony, G. Population-level analysis of gut microbiome variation. Science 2016, 352, 560-564. [CrossRef]

63. Wang, K.; Liao, M.; Zhou, N.; Bao, L.; Ma, K.; Zheng, Z.; Wang, Y.; Liu, C.; Wang, W.; Wang, J.; et al. Parabacteroides distasonis Alleviates Obesity and Metabolic Dysfunctions via Production of Succinate and Secondary Bile Acids. Cell Rep. 2019, 26, 222-235.e5. [CrossRef]

64. Koh, G.Y.; Kane, A.V.; Wu, X.; Xian Wu, J.W.C. Parabacteroides distasonis attenuates tumorigenesis, modulates inflammatory markers, and promotes intestinal barrier integrity in azoxymethane-treated A/J mice. Carcinogenesis 2020, 41, 909-917. [CrossRef]

65. Hiippala, K.; Kainulainen, V.; Suutarinen, M.; Heini, T.; Bowers, J.R.; Jasso-Selles, D.; Lemmer, D.; Valentine, M.; Barnes, R.; Engelthaler, D.M.; et al. Isolation of anti-inflammatory and epithelium reinforcing bacteroides and parabacteroides spp. From a healthy fecal donor. Nutrients 2020, 12, 935. [CrossRef]

66. Nguyen, M.; Vedantam, G. Mobile genetic elements in the genus Bacteroides, and their mechanism(s) of dissemination. Mob. Genet. Elements 2011, 1, 187-196. [CrossRef]

67. Biassoni, R.; Di Marco, E.; Squillario, M.; Barla, A.; Piccolo, G.; Ugolotti, E.; Gatti, C.; Minuto, N.; Patti, G.; Maghnie, M.; et al. Gut Microbiota In T1dm-Onset Pediatric Patients: Machine Learning Algorithms To Classify Microganisms Disease-Linked. J. Clin. Endocrinol. Metab. 2020, 105, 3114-3126. [CrossRef]

68. Roesch, L.F.W.; Lorca, G.L.; Casella, G.; Giongo, A.; Naranjo, A.; Pionzio, A.M.; Li, N.; Mai, V.; Wasserfall, C.H.; Schatz, D.; et al. Culture-independent identification of gut bacteria correlated with the onset of diabetes in a rat model. ISME J. 2009, 3, 536-548. [CrossRef]

69. Ma, Q.; Li, Y.; Wang, J.; Li, P.; Duan, Y.; Dai, H.; An, Y.; Cheng, L.; Wang, T.; Wang, C.; et al. Investigation of gut microbiome changes in type 1 diabetic mellitus rats based on high-throughput sequencing. Biomed. Pharmacother. 2020, 124, 109873. [CrossRef]

70. Yadav, R.; Dey, D.K.; Vij, R.; Meena, S.; Kapila, R.; Kapila, S. Evaluation of anti-diabetic attributes of Lactobacillus rhamnosus MTCC: 5957, Lactobacillus rhamnosus MTCC: 5897 and Lactobacillus fermentum MTCC: 5898 in streptozotocin induced diabetic rats. Microb. Pathog. 2018, 125, 454-462. [CrossRef] [PubMed]

71. Paone, P.; Cani, P.D. Mucus barrier, mucins and gut microbiota: The expected slimy partners? Gut 2020, 69, 2232-2243. [CrossRef] [PubMed]

72. Chassaing, B.; Raja, S.M.; Lewis, J.D.; Srinivasan, S.; Gewirtz, A.T. Colonic Microbiota Encroachment Correlates with Dysglycemia in Humans. Cell. Mol. Gastroenterol. Hepatol. 2017, 4, 205-221. [CrossRef]

73. Tsuchiya, K.; Kawamura, I.; Takahashi, A.; Nomura, T.; Kohda, C.; Mitsuyama, M. Listeriolysin O-induced membrane permeation mediates persistent interleukin-6 production in Caco-2 cells during Listeria monocytogenes infection in vitro. Infect. Immun. 2005, 73, 3869-3877. [CrossRef]

74. Nguyen, B.N.; Peterson, B.N.; Portnoy, D.A. Listeriolysin O: A phagosome-specific cytolysin revisited. Cell. Microbiol. 2019, 21, e12988. [CrossRef]

75. Cajnko, M.M.; Marušić, M.; Kisovec, M.; Rojko, N.; Benčina, M.; Caserman, S.; Anderluh, G. Listeriolysin O affects the permeability of Caco-2 monolayer in a pore-dependent and Ca2+-independent manner. PLoS ONE 2015, 10, e0130471. [CrossRef]

76. Zafar, H.; Saier, M.H. Comparative genomics of transport proteins in seven Bacteroides species. PLoS ONE 2018, 13 , e0208151. [CrossRef]

77. Richter, J.F.; Gitter, A.H.; Günzel, D.; Weiss, S.; Mohamed, W.; Chakraborty, T.; Fromm, M.; Schulzke, J.D. Listeriolysin O affects barrier function and induces chloride secretion in HT-29/B6 colon epithelial cells. Am. J. Physiol.Gastrointest. Liver Physiol. 2009, 296, 1350-1359. [CrossRef] [PubMed]

78. Ribet, D.; Cossart, P. How bacterial pathogens colonize their hosts and invade deeper tissues. Microbes Infect. 2015, 17, 173-183. [CrossRef]

79. Manfredo Vieira, S.; Hiltensperger, M.; Kumar, V.; Zegarra-Ruiz, D.; Dehner, C.; Khan, N.; Costa, F.R.C.; Tiniakou, E.; Greiling, T.; Ruff, W.; et al. Translocation of a gut pathobiont drives autoimmunity in mice and humans. Science 2018, 359, $1156-1161$. [CrossRef]

80. Wooldridge, L.; Ekeruche-Makinde, J.; Van Den Berg, H.A.; Skowera, A.; Miles, J.J.; Tan, M.P.; Dolton, G.; Clement, M.; LlewellynLacey, S.; Price, D.A.; et al. A single autoimmune T cell receptor recognizes more than a million different peptides. J. Biol. Chem. 2012, 287, 1168-1177. [CrossRef]

81. Coppieters, K.T.; Dotta, F.; Amirian, N.; Campbell, P.D.; Kay, T.W.H.; Atkinson, M.A.; Roep, B.O.; von Herrath, M.G. Demonstration of islet-autoreactive CD8 T cells in insulitic lesions from recent onset and long-term type 1 diabetes patients. J. Exp. Med. 2012, 209, 51-60. [CrossRef] [PubMed]

82. Fine, R.L.; Manfredo Vieira, S.; Gilmore, M.S.; Kriegel, M.A. Mechanisms and consequences of gut commensal translocation in chronic diseases. Gut Microbes 2020, 11, 217-230. [CrossRef] 
83. Abdelazez, A.; Abdelmotaal, H.; Evivie, S.E.; Melak, S.; Jia, F.F.; Khoso, M.H.; Zhu, Z.T.; Zhang, L.J.; Sami, R.; Meng, X.C. Screening potential probiotic characteristics of Lactobacillus brevis strains in vitro and intervention effect on type i diabetes in vivo. BioMed Res. Int. 2018, 2018. [CrossRef]

84. Teixeira, L.D.; Kling, D.N.; Lorca, G.L.; Gonzalez, C.F. Lactobacillus johnsonii N6.2 diminishes caspase-1 maturation in the gastrointestinal system of diabetes prone rats. Benef. Microbes 2018, 9, 527-539. [CrossRef]

85. Marques, T.M.; Patterson, E.; Wall, R.; O’Sullivan, O.; Fitzgerald, G.F.; Cotter, P.D.; Dinan, T.G.; Cryan, J.F.; Ross, R.P.; Stanton, C. Influence of GABA and GABA-producing Lactobacillus brevis DPC 6108 on the development of diabetes in a streptozotocin rat model. Benef. Microbes 2016, 7, 409-420. [CrossRef]

86. Kim, T.K.; Lee, J.C.; Im, S.H.; Lee, M.S. Amelioration of Autoimmune Diabetes of NOD Mice by Immunomodulating Probiotics. Front. Immunol. 2020, 11, 1832. [CrossRef]

87. Putt, K.K.; Pei, R.; White, H.M.; Bolling, B.W. Yogurt inhibits intestinal barrier dysfunction in Caco-2 cells by increasing tight junctions. Food Funct. 2017, 8, 406-414. [CrossRef] [PubMed]

88. Monteagudo-Mera, A.; Rastall, R.A.; Gibson, G.R.; Charalampopoulos, D.; Chatzifragkou, A. Adhesion mechanisms mediated by probiotics and prebiotics and their potential impact on human health. Appl. Microbiol. Biotechnol. 2019, 103, 6463-6472. [CrossRef]

89. Jung, S.H.; Hong, D.K.; Bang, S.J.; Heo, K.; Sim, J.J.; Lee, J.L. The functional properties of Lactobacillus casei hy2782 are affected by the fermentation time. Appl. Sci. 2021, 11, 2481. [CrossRef]

90. Taddese, R.; Garza, D.R.; Ruiter, L.N.; de Jonge, M.I.; Belzer, C.; Aalvink, S.; Nagtegaal, I.D.; Dutilh, B.E.; Boleij, A. Growth rate alterations of human colorectal cancer cells by 157 gut bacteria. Gut Microbes 2020, 12, 1-20. [CrossRef]

91. Wexler, A.G.; Schofield, W.B.; Degnan, P.H.; Folta-Stogniew, E.; Barry, N.A.; Goodman, A.L. Human gut Bacteroides capture vitamin B12 via cell surface-exposed lipoproteins. Elife 2018, 7, e37138. [CrossRef] 